Abstract Plasmon lasers are a new class of coherent optical frequency electromagnetic wave amplifiers that deliver intense, coherent and directional surface plasmons well below the diffraction barrier. The strongly confined electric fields in plasmon lasers can enhance significantly light-matter interactions and bring fundamentally new capabilities to bio-sensing, data storage, photolithography and optical communications.

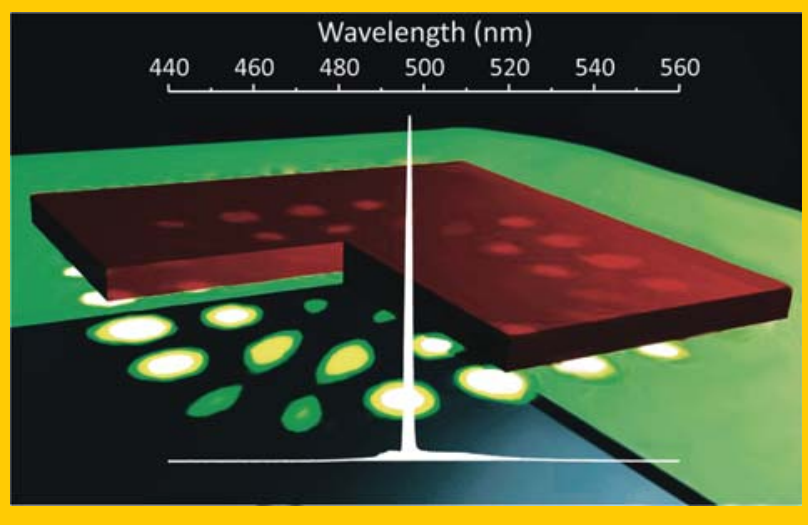

\title{
Plasmon lasers: coherent light source at molecular scales
}

\author{
Ren-Min Ma ${ }^{1}$, Rupert F. Oulton ${ }^{1,3}$, Volker J. Sorger ${ }^{1}$, and Xiang Zhang ${ }^{1,2,{ }^{*}}$
}

\section{Introduction}

Lasers are the brightest sources of high frequency electromagnetic radiation with applications spanning all physical sciences and engineering. Since the first demonstration over 50 years ago [1], laser science has seen tremendous developments of high intensity lasers for nuclear fusion [2,3], faster lasers for probing sub-femtosecond chemical reactions and atomic processes $[4,5]$ and smaller lasers for large scale integrated optical data networks that form the backbone of the internet [6]. The study of the emerging field of nanophotonics now calls for coherent light sources free from diffraction limitations as a new powerful tool to investigate the rich physics at the nanometer scale and develop high performance devices with new capabilities. Recently, a new class of optical amplification and laser action was demonstrated for charge density waves at the nanoscale, named plasmon lasers or spaser [7-15]. In contrast to classical lasers, plasmon lasers amplify light coupled to oscillating electrons, which adds momentum to light enabling their physical size and mode volume to shrink below the diffraction limit. The minimum physical size of a plasmon laser can be just a few nanometers, limited only by a metal's inhomogeneity and non-locality length scale $(\sim 1 \mathrm{~nm})$ [16]. With unprecedented small physical size and mode volume, new features of plasmon laser are evident. Firstly, the strong electromagnetic confinement in a plasmon cavity modifies the laser action by enhancing spontaneous emission [9,11,17-19]. This causes a drastic spatial redistribution of spontaneous emission. That is to say, light can be inhibited into some modes and preferentially coupled to the laser mode. This can significantly reduce the pump conditions for the onset of laser action and will modify the threshold behavior. Secondly, the electron collision time within metals is of the order of 100 femtoseconds, leading to the high Ohmic loss of the plasmon cavity. This can affect a number of processes within a laser, but most importantly, due to this short plasmon lifetime and the high Purcell factor, plasmon lasers can be potentially modulated at frequencies in the terahertz range [16,20].

With the ability to generate high intensity electromagnetic radiation at the nanoscale in femtosecond timescales, the recent emergence of plasmon lasers now stimulates the exploration of nanometer-scale science and application towards the rich physics of deep sub-wavelength optics and the development of high performance devices with sizes comparable to that of viruses (or a transistor inside a CPU). For instance, the highly focused near field of plasmon lasers could be used for photolithography, enabling feature sizes beyond the limits of conventional lasers [21-23]. They could also be useful for packing more data onto storage media such as DVDs or hard disks [24,25]. Moreover, the strong electric field confinement in plasmon lasers could be utilized to amplify very weak effects. This could be deployed for detecting single molecules, allowing for extremely sensitive bio-detection and in-situ nanoscale Raman and Fluorescence spectroscopy [26-31]. Furthermore, owing to their small size and potentially fast operation speed, they are a promising light source for ultra-compact and fast photonic circuits.

In this review, we will first discuss the general approaches to construct plasmon lasers and the key realizations of experimental plasmon laser demonstrations. Then we will discuss the fundamental physics and operational characteristics. In particular, we review how the plasmonic confinement effect influences plasmon laser action through the

\footnotetext{
1 NSF Nanoscale Science and Engineering Centre, 3112 Etcheverry Hall, University of California, Berkeley, CA 94720, USA

2 Materials Sciences Division, Lawrence Berkeley National Laboratory, 1 Cyclotron Road, Berkeley, CA 94720, USA

3 Experimental Solid State Physics, The Blackett Laboratory, Imperial College London, London SW7 2AZ, UK

Corresponding author: e-mail: xiang@berkeley.edu
} 
enhancement of spontaneous emission in sub-wavelength scale plasmonic cavities as well as the ultrafast behavior of plasmon laser. Finally, we discuss future prospects and challenges for the field.

\section{Recent progress of plasmon lasers}

\subsection{Theoretical considerations of a plasmon laser}

Nanoscale optical fields excited on metallic nanostructures strongly enhance optical intensities and have a wide range of applications; however, the resonant excitation of these local fields by "nano-focusing" an external optical field has a limited coupling efficiency [32-38] and can only access "bright modes" that couple to the far-field. Meanwhile, "dark" modes, which couple only very weakly to the far-field, usually exhibit the strongest confinement. In 2003, Bergman and Stockman proposed that by lasing into localized surface plasmons, a coherent light field can be generated directly at the nano-scale [39]. This essentially allows focusing of optical energy beyond the diffraction limit in a much more efficient and compact way compared to passive nano-focusing. While the stimulated emission of surface plasmons were observed by several groups [40,41], it wasn't until 2009 while the first plasmon lasers were experimentally realized. Three groups reported plasmon lasers in three different configurations within three months of each other. Furthermore, each group demonstrated a plasmon laser exhibiting subwavelength confinement in a different number of dimensions (Fig. 1). Hill and co-workers reported an elongated pillar structure defining two closely spaced metal walls sandwiching a gain medium [7]. This so called Metal-Insulator-Metal (MIM) waveguide-cavity provides plasmonic confinement in one dimension between the metal walls by supporting a confined waveguide mode that overlaps with the III-V (InGaAs) gain media. The mode's reflections at the metalcoated waveguide end facets defined a Fabry-Perot cavity to provide feedback for the laser. Later, Oulton et al. reported a hybrid nanowire Metal-Insulator-Semiconductor
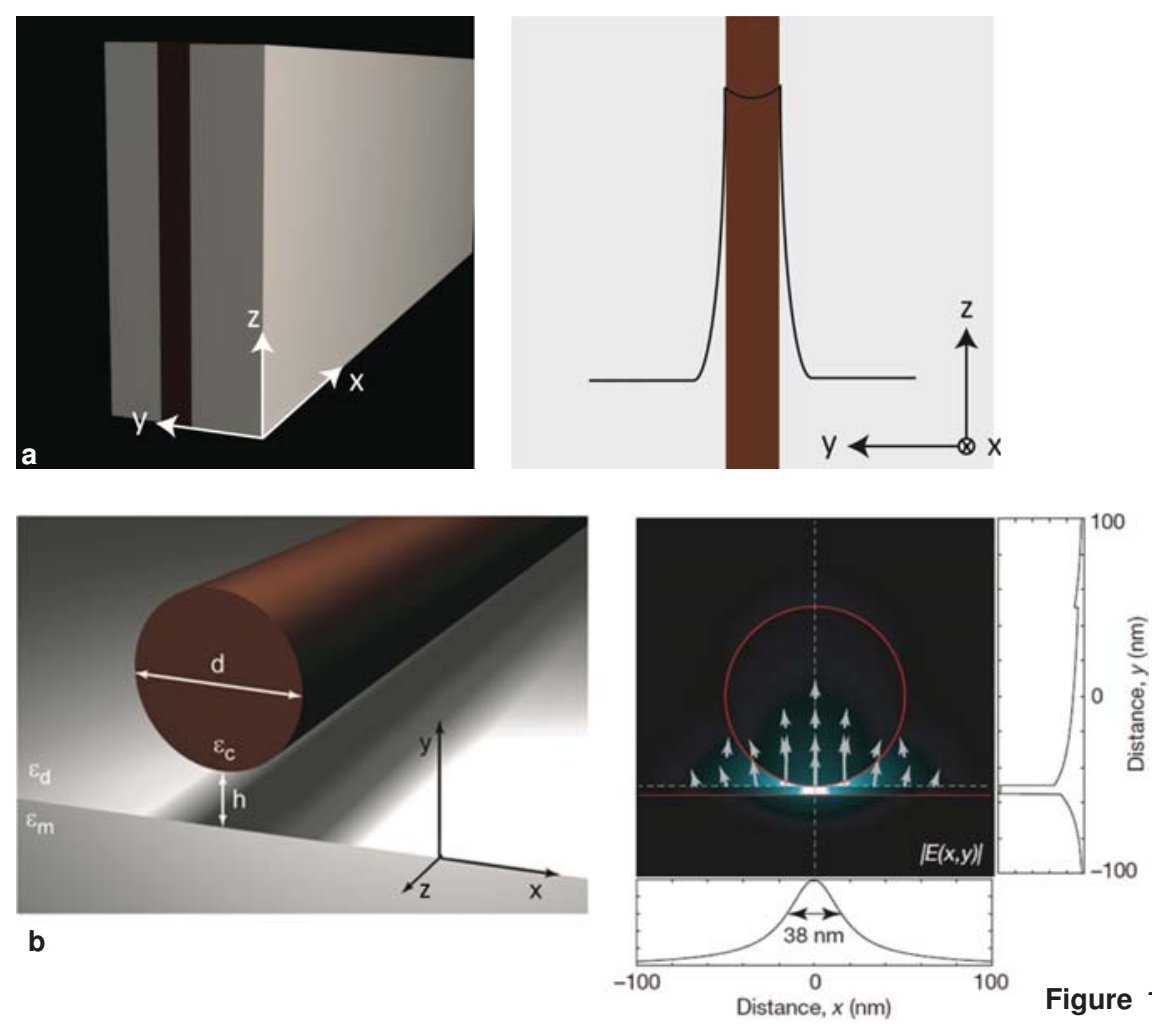

(online color at: www.lpr-journal.org) The first demonstrated plasmon lasers exhibiting
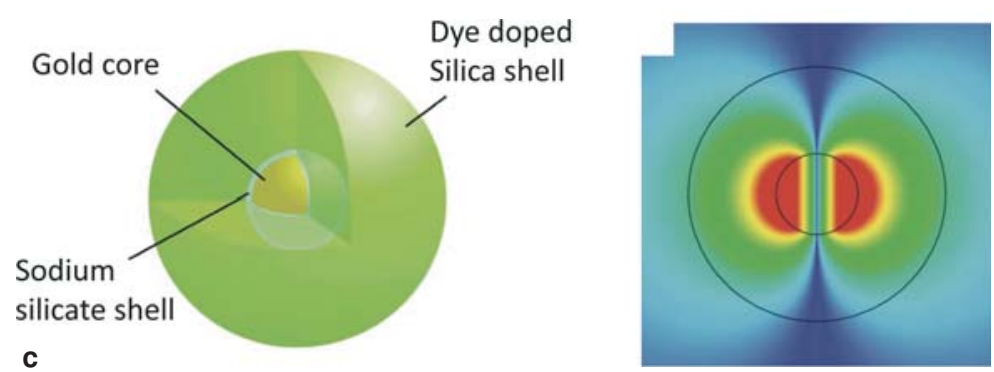
sub-wavelength confinement in a various numbers of dimensions. a) Plasmon laser based on MetalInsulator-Metal waveguide-cavity provides plasmonic confinement in one dimension. b) The MetalInsulator-semiconductor hybrid design achieves strong optical confinement in the insulating gap region in two dimensions. c) The plasmon laser based on localized surface plasmon dipole like cavity mode providing confinement in all three spatial dimensions. 
(MIS) plasmon laser [9]. The MIS hybrid design achieves strong optical confinement in the insulating gap region (few nanometers thick) in two dimensions. The two end facets of the nanowire serve as mirrors to form a Fabry-Perot cavity. Noginov and co-workers reported a localized surface plasmon laser comprised of a dielectric clad spherical gold particle measuring just $44 \mathrm{~nm}$ in diameter [8]. Here the $15 \mathrm{~nm}$ diameter gold particle supports a dipole-like plasmonic cavity mode, while a Silica shell impregnated with OG-488 dye molecules serves as the gain medium. The dipole-like nature of the resonance provides plasmonic confinement in all 3 spatial dimensions. While, varying in confinement dimensionality, in all three cases the plasmonic laser modes cannot be efficiently excited by resonant coupling from the far-field; efficient excitation is only possible by laser action directly into the plasmonic modes. These plasmon lasers use the two types of surface plasmon cavity: propagating surface plasmon polaritons resonating between two mirrors and localized surface plasmon. Here we will discuss these two approaches and the plasmon lasers based on them briefly.

\subsubsection{Plasmon lasers based on propagating surface plasmon polaritons}

Surface plasmon polaritons (SPPs) are electromagnetic excitations propagating at the interface of a dielectric and a conductor, evanescently confined in the direction perpendicular to the interface. As a simple example, we discuss a geometry sustaining SPPs of a single, flat interface between a dielectric with positive real dielectric constant $\varepsilon_{2}$ and an adjacent conducting half space described via a dielectric function $\varepsilon_{1}(\omega)$ with $\operatorname{Re}\left[\varepsilon_{1}\right]<0$. By solving Maxwell's equations for the electromagnetic wave at such an interface with the boundary conditions, the dispersion relation of SPPs propagating at the interface can be described as, $\beta=k_{0} \sqrt{\frac{\varepsilon_{1} \varepsilon_{2}}{\varepsilon_{1}+\varepsilon_{2}}}$, where $k_{0}$ is the wave vector of the propagating wave in vacuum and $\beta$ is the propagation constant of the traveling waves and corresponds to the component of the wave vector in the direction of propagation [42]. This dispersion curve lies to the right of the light line of the corresponding dielectric due to the nature of dispersion of metal permittivity and approaches the characteristic surface plasmon frequency, $\omega_{s p}=\frac{\omega_{p}}{\sqrt{1+\varepsilon_{2}}}$, where $\omega_{p}$ is the plasmon frequency of conduction electrons (Fig. 2a). This unique dispersion relation enables SPPs with large propagating wave vectors comparing to that of the light wave propagating in the pure dielectric layer. SPPs at frequencies closer to $\omega_{s p}$ exhibit larger field confinement to the interface leading to strong confinement of the light field to a fraction of their free space wavelength. This larger confinement is accompanied by the shorter propagation distance due to increased damping. There are various waveguide configurations supporting propagating SPPs, such as dielectric-loaded [43-45], nanowire [46], wedge and groove [47,48], MIM [49,50] and hybrid MIS plasmon waveguides [51-53]. Plasmon lasers using propagating SPPs can be constructed based on these plasmon waveguides by replacing the dielectric layer in these waveguide using gain materials such as direct band gap semiconductors and introducing some feedback mechanism such as Fabry-Perot [7,9,12], distributed feed-back [13,14] and total internal reflection whispering gallery $[10,11,15]$ cavities. Since propagating SPPs are not based on any resonant mechanism, they provide broadband confinement and the choice of gain materials is very flexible.

\subsubsection{Plasmon lasers based on localized surface plasmons}

Localized surface plasmons are non-propagating excitations of the conduction electrons of metallic nanoparticles coupled to the electromagnetic field (Fig. 2b). When the sizes of the metallic nanoparticles are much shorter than the excited wavelength in free space, the interaction of the metallic nanoparticles with the electromagnetic field can be analyzed using a simple quasi-static approximation, since the phase of the harmonically oscillating electromagnetic field is approximately constant over the particle volume. Then the spatial field distribution can be calculated by assuming the nanostructure in an electrostatic field and adding the harmonic time dependence to the solution later. The solution of Laplace's equation with boundary conditions predicts that the nanostructure can act as a resonant electric dipole surface plasmon mode due to a resonance of the polarizability. For instance, the resonant frequency of a perfect metal nanosphere with permittivity $\varepsilon_{1}$ embedded in surrounding dielectric with permittivity $\varepsilon_{2}$ is given by $\operatorname{Re}\left[\varepsilon_{1}\right]=-2 \varepsilon_{2}$ [42]. For non-regular shaped particles, the resonant frequency not only depends on the permittivity of the surrounding dielectric environment but also the shape, which supplies the flexibility for choosing materials with gain spectra overlapped with the resonant frequency. Note that this quasi-static approximation is strictly valid only for vanishingly small particles. For larger particles, the phase of the driving field changes over the particle volume and

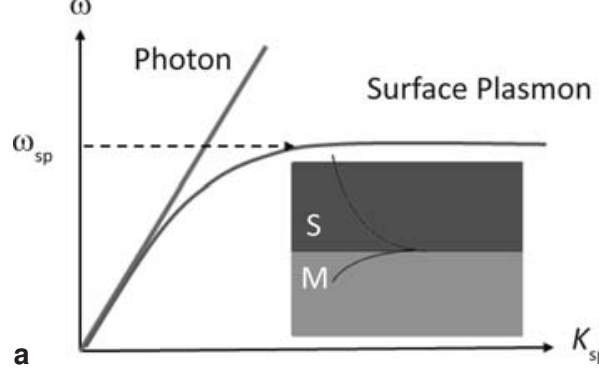

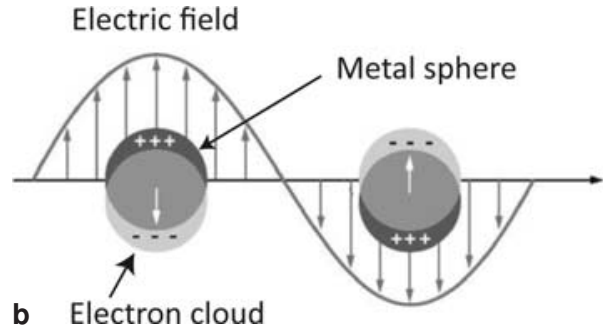

Figure 2 a) The dispersion relation of SPPs propagating at the interface of a metal and a dielectric. b) Localized surface plasmons of spherical metal nanoparticles oscillating with the electromagnetic field. 
Mie theory must be employed. Plasmon lasers using the localized surface plasmon modes can be constructed based on these metal nanoparticles by replacing the dielectric surrounding layer to gain materials where the particle itself can serve as a resonant cavity

Though we discuss propagating and localized surface plasmons separately, there is no clear boundary between them. Firstly, when the frequency of propagating SPPs approaches $\omega_{s p}$, the group velocity of the electromagnetic wave tends to zero as well as the phase velocity and the field becomes more confined, so that the propagating SPPs resemble localized surface plasmons. Secondly, for extremely strong cavity feedback of SPPs, radiative scattering from the cavity is almost completely suppressed, similar to the localized surface plasmons of small metal particles. However, stronger optical confinement also comes with a trade-off; that is the higher ohmic loss due to electron collisions in the metal. Though it is quite challenging to compensate the high ohmic loss of confined SPPs using known gain materials, the scaling down of plasmon lasers is attractive since their physical size can be comparable to the transistor in microelectronic technology but potentially much faster.

\subsection{Experimental demonstration of plasmon lasers}

We have introduced some of the general configurations to construct plasmon lasers above. In the following, we discuss several representative examples of experimentally demonstrated plasmon lasers and their properties in more detail. The devices we have chosen to focus on here are representative of the various SPP confinement and feedback mechanisms employed in the literature. We also review recent literature on metal lasers based on non-plasmonic modes highlighting their practical significance.

\subsubsection{One dimensional confinement}

\section{Fabry-Perot cavity}

The first plasmon laser utilized a MIM propagating SPP waveguide configuration (Fig. 3) [7]. This MIM waveguide features a confined propagating $\mathrm{TM}_{01}$ mode that overlaps with the III-V (InGaAs) gain media. The mode's reflections at the end of the waveguide formed a Fabry-Perot cavity to provide feedback for the laser. Due to the long cavity length (3-6 $\mu \mathrm{m})$, the cavity $Q$-factors of 370 (140) can be reached at cryogenic (room) temperatures, which also depends on the gain-core thickness. Electron beam lithography, lift off and reactive-ion-etching were used to define the semiconductor wall as the gain core, followed by coating the pillar wall by a $20 \mathrm{~nm}$ thick insulating silicon nitride and a silver cladding layer. This insulating layer was important to prevent optical quenching from the metal. The index contrast of the InGaAs gain layer and InP layers confines the light vertically in the waveguide. The plasmonic nature of this laser was confirmed by its sub-wavelength gain media thickness and observing an increase of the lasing mode's group index with shrinking wall width. For the thinnest reported device (width $=90 \mathrm{~nm}$ ) the measured group index was three times higher as compared to wide devices ( width $=300 \mathrm{~nm}$ ) suggestive of its plasmonic nature. In order to obtain an agreement between the observed group index and simulation results, an increasing dispersion with decreasing wall/waveguide width had to be assumed, which is consistent with increase in gain necessary to compensate the higher losses of more confined SPPs. The thin plasmonic MIM waveguide laser required cryogenic temperatures for lasing operation, while the wider example allows for room temperature operation, due to the available photonic mode with much lower Ohmic loss.

\section{Distributed feed-back cavities}

Hill's group has also investigated MIM SPP waveguides with vertical groove Bragg gratings as feedback mirrors [13]. These metallic Bragg gratings can provide a broad bandwidth stop-band $(\sim 500 \mathrm{~nm})$ with extremely high grating coupling coefficients. They showed that the spontaneous emission within the stop-bands of these Bragg grating cavities can be strongly suppressed, which can significantly reduce lasing thresholds when compared to similar length Fabry-Pérot waveguide cavities. At $80 \mathrm{~K}$, they observe a clear lasing signal in a device with total length of about $100 \mu \mathrm{m}$ under DC electrical pump. The light emission from the device was strongly TM polarized, which is consistent with the MIM SPP gap mode. Though the device cannot work at room temperature, they observed strong linewidth

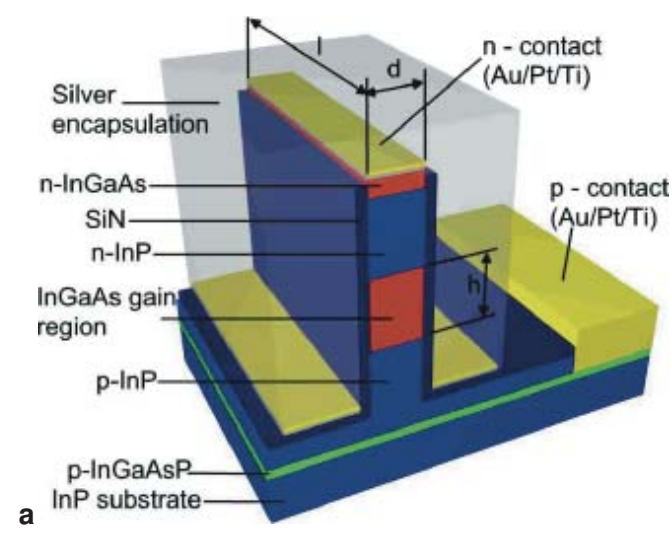

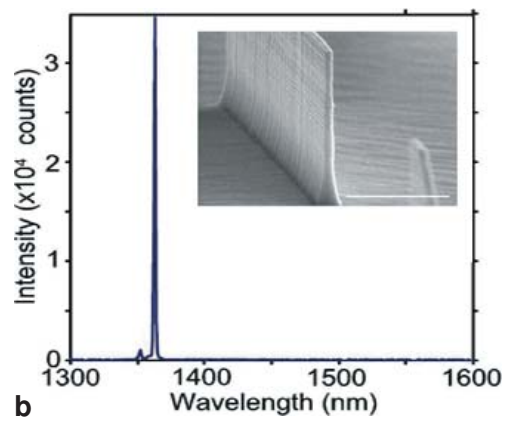

Figure 3 (online color at: www.lprjournal.org) Metal-insulator-Metal (MIM) laser. a) Schematic of the cavity formed by a rectangular semiconductor pillar encapsulated in Silver. b) Above threshold emission spectrum for 6 micron long device with semiconductor core with width of $90 \mathrm{~nm}$, with pump current $200 \mu \mathrm{A}$ at $10 \mathrm{~K}$. Inset: Scanning electron microscope image showing the semiconductor core of one of the devices. The scale bar is 1 micron. From [7]. 
reduction and a super-linear light current curve under pulsed pump at $296 \mathrm{~K}$. Such devices are electrically pumped, which is critical for most practical application.

In a related approach to generate strong SPP feedback, Lakhani et al. reported a nanolaser based on a bandgap defect state inside a surface plasmonic crystal [14]. The devices operated under pulsed optical pumping at $77 \mathrm{~K}$.

\section{Whispering gallery cavity}

Another 1-D mode confined plasmon nanolaser design by Kwon et al. utilizes a confined whispering-gallery cavity at a semiconductor-metal interface in a configuration of InP/InAsP/InP disk coated by silver [10]. Plasmonic confinement is achieved in 1 dimension in this design. In this geometry lasing was observed up to liquid nitrogen temperatures upon optically pumping the device. The $235 \mathrm{~nm}$ thick $\mathrm{InP} / \mathrm{InAsP} / \mathrm{InP}$ disk were formed on the glass substrate using electron-beam lithography and a dry etching process. After that, silver was evaporated on the disk to form a pan-shaped metal coated cavity. The whispering-gallery plasmon mode can lase under optical pumping with increasing threshold from $8 \mathrm{~K}$ to $80 \mathrm{~K}$ while no lasing was observed at $300 \mathrm{~K}$.
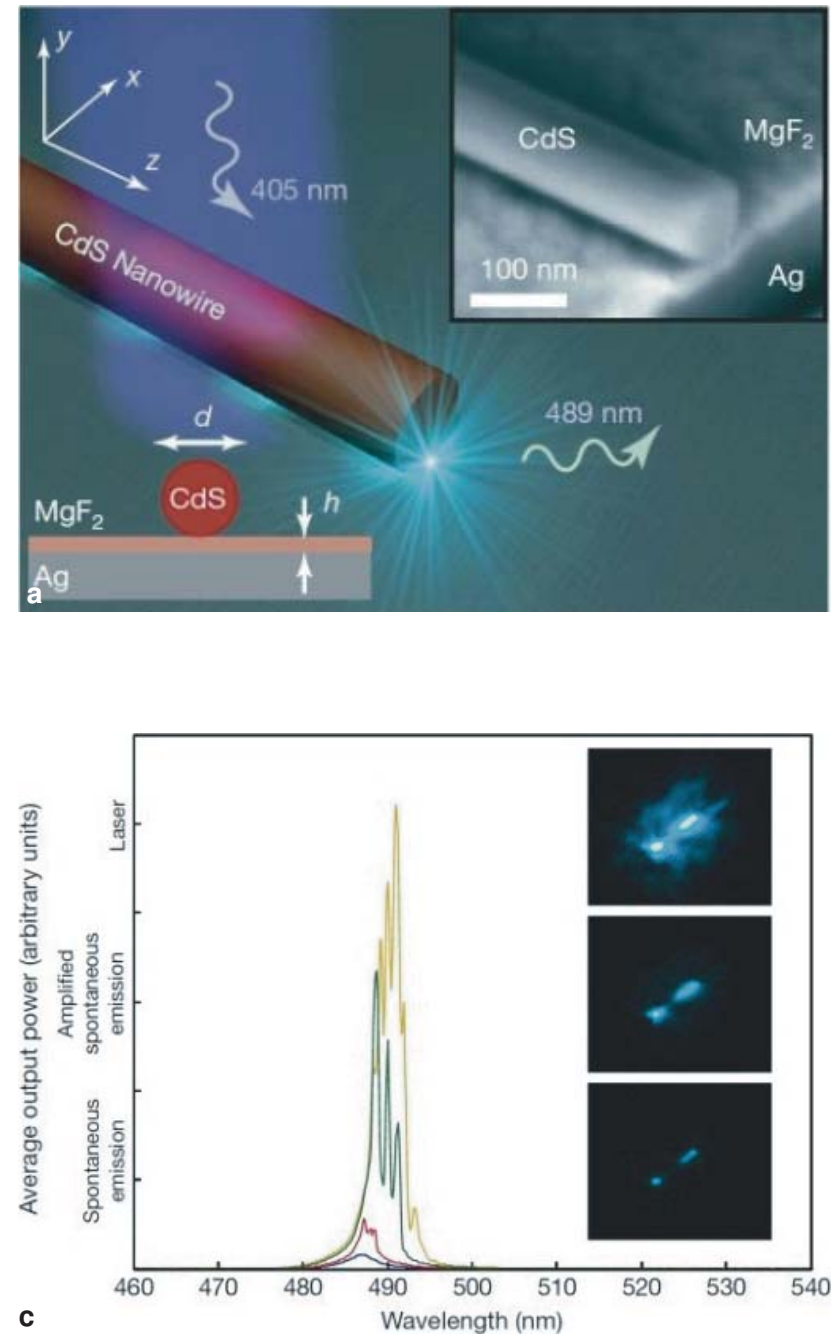

\subsubsection{Two dimensional confinement}

\section{Hybrid MIS Fabry-Perot type cavity}

A plasmonic laser exhibiting two dimensional plasmonic confinements was realized in a hybrid MIS nanowire structure (Fig. 4) [9]. Key to this design was the high permittivity contrast at the low/high-dielectric interfaces leading to a strong optical confinement in a thin (few nanometer) gap region. This creates a 2D confined hybrid-plasmon-polariton (HPP) mode with a broadband, deep sub-wavelength mode size and relatively low propagation loss (Fig. 4b) [9,49]. For the purpose of demonstrating a plasmon laser the authors used a semiconductor $(\mathrm{CdS})$ nanowire $[54,55]$, which effectively served three functions at once; the gain media, part of the confinement mechanism and forms the cavity feedback via the high index contrast inside the laser and air. The plasmonic character of this laser was confirmed in a number of ways: firstly, a strong polarization dependence and enhanced spontaneous emission factors corresponding to the plasmonic mode were reported (Purcell factor $=6$ ). Furthermore, the plasmon laser could operate for smaller nanowire diameters than photonic nanowire lasers, due to the strong

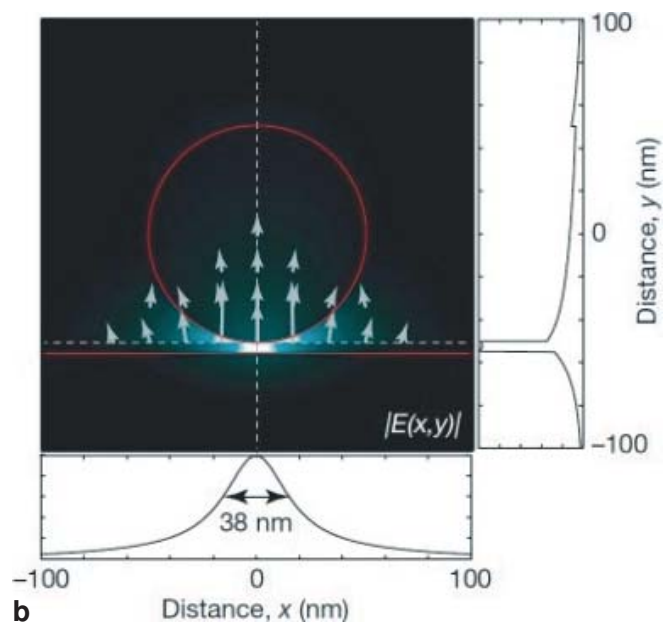

Figure 4 (online color at: www.lpr-journal.org) Waveguide Semiconductor Plasmon Nanolaser. a) This plasmonic laser consists of a CdS semiconductor nanowire on top of a silver substrate, separated by a nanometer-scale $\mathrm{MgF}_{2}$ layer of thickness $\mathrm{h}$. The inset shows a scanning electron microscope image of a typical plasmonic laser. b) The stimulated electric field distribution and direction $|E(x, y)|$ of a hybrid plasmonic mode at a wavelength of $489 \mathrm{~nm}$. The cross-sectional field plots (along the broken lines in the field map) illustrate the strong overall confinement in the gap region between the nanowire and metal surface with sufficient modal overlap in the semiconductor to facilitate gain. c) Laser oscillation of a plasmonic laser, $d=129 \mathrm{~nm}, h=5 \mathrm{~nm}$. The four spectra for different peak pump intensities exemplify the transition from spontaneous emission $\left(21.25 \mathrm{MW} \mathrm{cm}^{-2}\right)$ via amplified spontaneous emission $\left(32.50 \mathrm{MW} \mathrm{cm}^{-2}\right)$ to full laser oscillation ( $76.25 \mathrm{MW} \mathrm{cm}^{-2}$ and $131.25 \mathrm{MW} \mathrm{cm}^{-2}$ ) at $10 \mathrm{~K}$. Inset: corresponding far field images of the waveguide plasmon nanolaser with increasing pump (bottom to top). From [9]. 
confinement surpassing the diffraction limit. Under optical pumping, lasing was observed for plasmonic nanowire lasers with diameters of just $52 \mathrm{~nm}$, while photonic lasers reached a cut-off for nanowire diameters approaching $140 \mathrm{~nm}$.

\subsubsection{Three dimensional plasmonic confinement}

The physical size of a plasmonic laser is linked to the smallest possible surface plasmon cavity; since metallic nanoparticles down to a few nanometer in diameter can still support a localized surface plasmon [16], in principle, plasmonic lasers could have similar dimensions. Indeed, Noginov and co-workers have reported such a nanoparticle laser comprised of a dielectric clad spherical gold particle measuring just $44 \mathrm{~nm}$ in diameter [8]. Here the $15 \mathrm{~nm}$ diameter gold particle supports a dipole like plasmonic cavity mode while a Silica shell impregnated with OG-488 dye molecules serves as the gain medium (Fig. 5). The authors report the signature of laser action under strong optical excitation with threshold pump pulse energies in the milli-Joule range. However, out of all of the plasmonic lasers reported to date, this is the only configuration that is capable of reaching the limits of plasmonics determined by the metal's non-locality; but has yet to see a follow-up demonstration. Since there is a growing interest in the use of gain with arrays of such plasmonic nanoparticles towards low-loss metamaterials [56-59], the laser action of metallic nanoparticles has become a very important research area. In the following summary we raise some fascinating questions that arise from this work, which point to possible research directions. The first one is the radiation and non-radiation ratio. The plasmon lasers based on localized surface plasmons have intrinsic feedback due to the plasmon resonance. Furthermore, the devices deliver en- ergy to the nanoscale plasmonic mode but just release only a small part of their optical energy to the far field before it is dissipated in the metal. For such a small metal particle, the absorption lifetime is about $25 \mathrm{fs}$ (assuming a quality factor of 15 at $\lambda=532 \mathrm{~nm}$ ), while the radiative lifetime is 8 ps estimated by Wheeler-Chu limit [60]; only $0.3 \%$ of generated surface plasmons couple out into the far field. We will discuss this issue in Sect. 4 in more detail. The characteristic lifetimes of this nano-particle laser further point to the question of whether laser action is actually achieved in the device. While Noginov and co-workers clearly see the characteristic kink indicating a transition from spontaneous to stimulated emission dominated processes, the observed lineshape is inconsistent with current spaser theory, which predicts much narrower linewidths near threshold $[16,38]$. Here, the reader should note that all plasmonic lasers reported to date also exhibit much broader linewidths than conventional lasers; however, in this particular work, the observed laser linewidth suggests a gain-compensated cavity lifetime of about $200 \mathrm{fs}$, which is $7 \mathrm{x}$ larger than the internal plasmonic losses, but considerably shorter than the estimated 8 ps radiative lifetime of the plasmonic cavity. It is clear that some reassessment of threshold and coherence is appropriate for plasmonic lasers. The scope for future applications for this type of device is currently unclear. The high optical pumping thresholds are inhibitive and mainly due to the extremely small optical cross section of each device. This could be reduced by electrical injection, where a semiconductor gain material would be preferable to the organic one here. Nevertheless, this demonstration shows us what we can expect from reducing the size of laser devices beyond the diffraction limit.
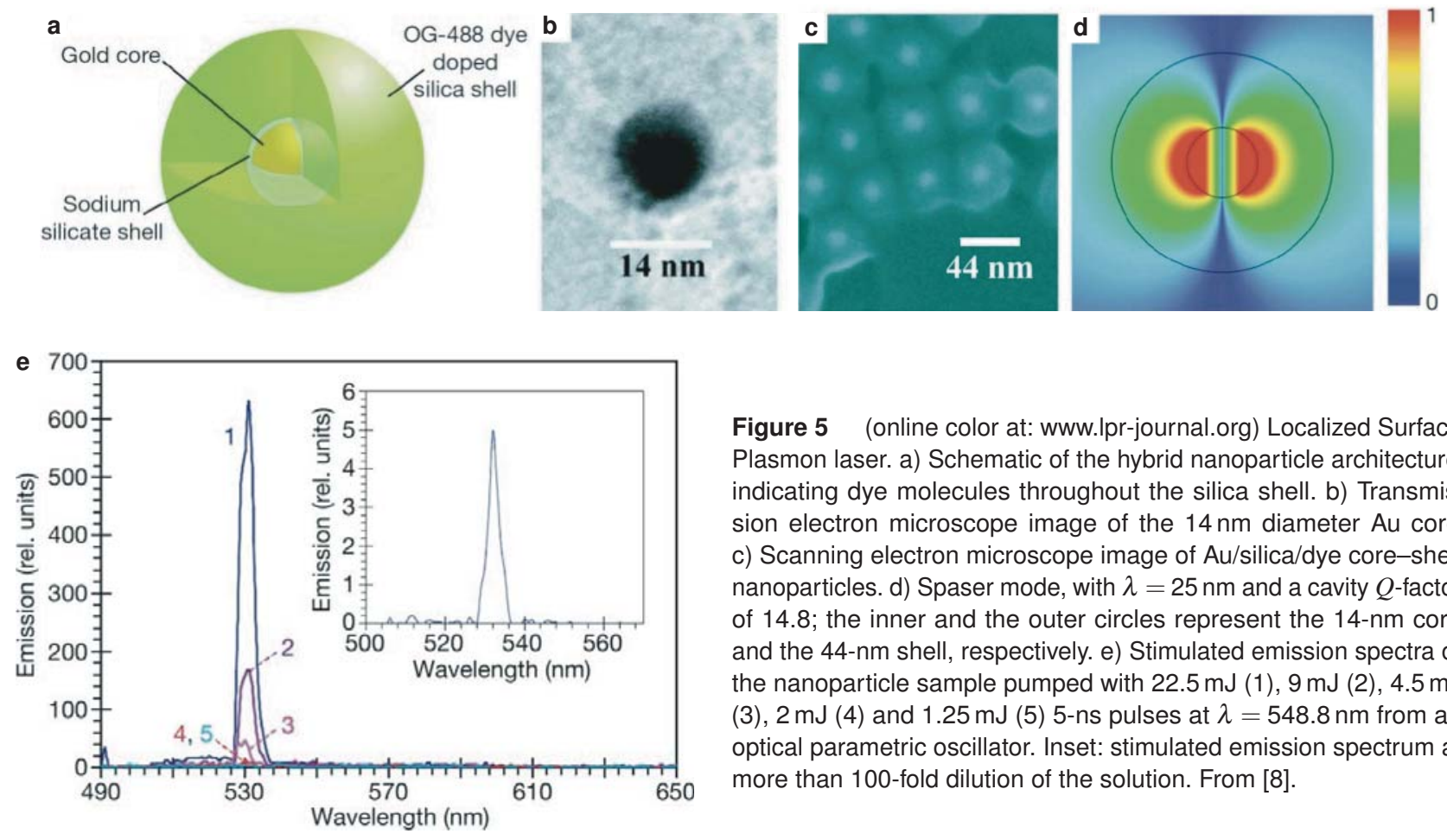

Figure 5 (online color at: www.lpr-journal.org) Localized Surface Plasmon laser. a) Schematic of the hybrid nanoparticle architecture, indicating dye molecules throughout the silica shell. b) Transmission electron microscope image of the $14 \mathrm{~nm}$ diameter Au core. c) Scanning electron microscope image of $\mathrm{Au} / \mathrm{silica} / \mathrm{dye}$ core-shell nanoparticles. d) Spaser mode, with $\lambda=25 \mathrm{~nm}$ and a cavity $Q$-factor of 14.8; the inner and the outer circles represent the 14-nm core and the 44-nm shell, respectively. e) Stimulated emission spectra of the nanoparticle sample pumped with $22.5 \mathrm{~mJ}(1), 9 \mathrm{~mJ}(2), 4.5 \mathrm{~mJ}$ (3), $2 \mathrm{~mJ}$ (4) and $1.25 \mathrm{~mJ}$ (5) 5 -ns pulses at $\lambda=548.8 \mathrm{~nm}$ from an optical parametric oscillator. Inset: stimulated emission spectrum at more than 100 -fold dilution of the solution. From [8]. 

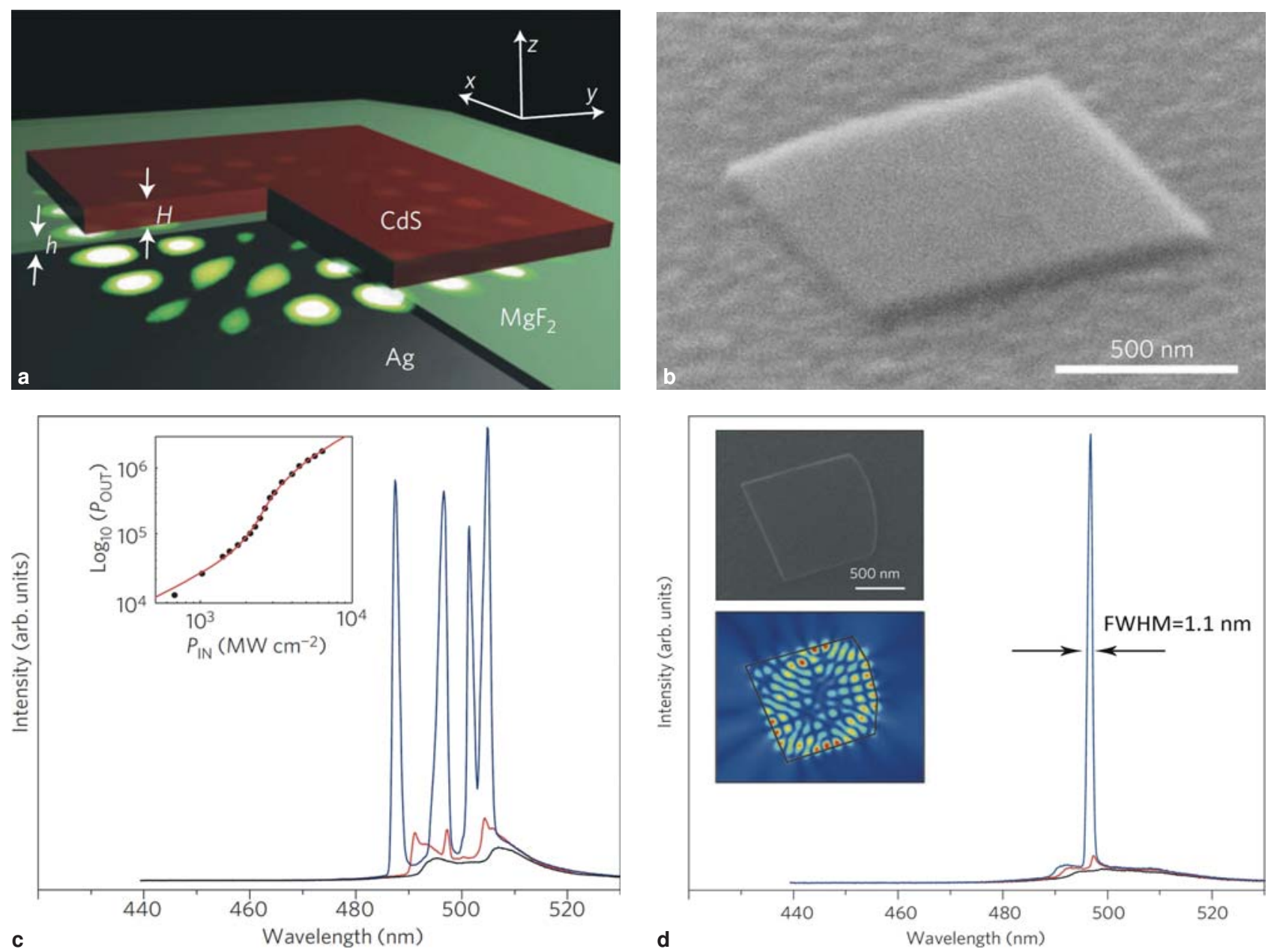

Figure 6 (online color at: www.lpr-journal.org) Room-temperature hybrid plasmon nanolaser. a) Schematic of the room-temperature plasmon laser showing a thin CdS square atop a silver substrate separated by a $5 \mathrm{~nm} \mathrm{MgF} 2$ gap, where the most intense electric fields of the device reside. b) SEM micrograph of a $45 \mathrm{~nm}$ thick, $1 \mu \mathrm{m}$ length CdS square plasmon laser. c) Room-temperature laser spectra and integrated light-pump response (inset) of the laser in panel b) showing the transition from spontaneous emission $\left(1,960 \mathrm{MW} \mathrm{cm}^{2}\right.$, black) through amplified spontaneous emission $\left(2,300 \mathrm{MW} \mathrm{cm}^{2}\right.$, red) to full laser oscillation $\left(3,074 \mathrm{MW} \mathrm{cm}^{2}\right.$, blue). d) Laser spectrum and integrated light-pump response of a single-mode room-temperature plasmon laser. Inset: top, the SEM micrograph of the device. The curved facet breaks the four-fold symmetry and thereby inhibits high-order modes that exist in the square CdS lasers. Bottom, the electric-field-intensity distribution of the lasing mode. From [11].

\subsubsection{Room temperature operation}

Currently, the plasmon lasers using inorganic semiconductors as gain materials are the most technologically relevant being capable of electrical injection and compatible with the current fabrication processes of computing and communications infrastructure. Furthermore, semiconductor materials are robust giving such laser devices long lifetimes. However, the cryogenic temperature operation of the semiconductor plasmon lasers is a major hurdle towards their practical application. Room temperature plasmon laser operation below the diffraction limit demands low metal loss, effective cavity feedback and high gain; all within a single nanoscale device. Recently, Ma et al. reported a room temperature semiconductor plasmon laser (Fig. 6) [11]. Here, total internal reflection of SPPs is adopted to mitigate the radiation loss, while utilizing the hybrid MIS [51] design in a nano-squares cavity configuration. This laser design features strong confinement while simultaneously having low metal and radiation loss enabling its room temperature operation and a much shorter cavity length compared to those reported at cryogenic temperatures. The device consists of a $45 \mathrm{~nm}$ thick, $1 \mu \mathrm{m}$ length single crystal $\mathrm{CdS}$ square $[61,62]$ atop a Silver surface separated by a $5 \mathrm{~nm}$ thick Magnesium Fluoride gap layer, shown in Fig. 6b,c. Strong feedback was achieved by total internal reflection of surface plasmons at the cavity boundaries resulting in sufficiently high quality factors that can be identified by pronounced cavity modes even in the spontaneous emission spectrum far below laser threshold in Fig. 6c. This feedback mechanism only establishes plasmonic cavity modes since photonic modes of the semiconductor slab have insufficient momentum for total internal reflection. Furthermore, lasing in such ultrathin devices is viable solely due the plasmonic 

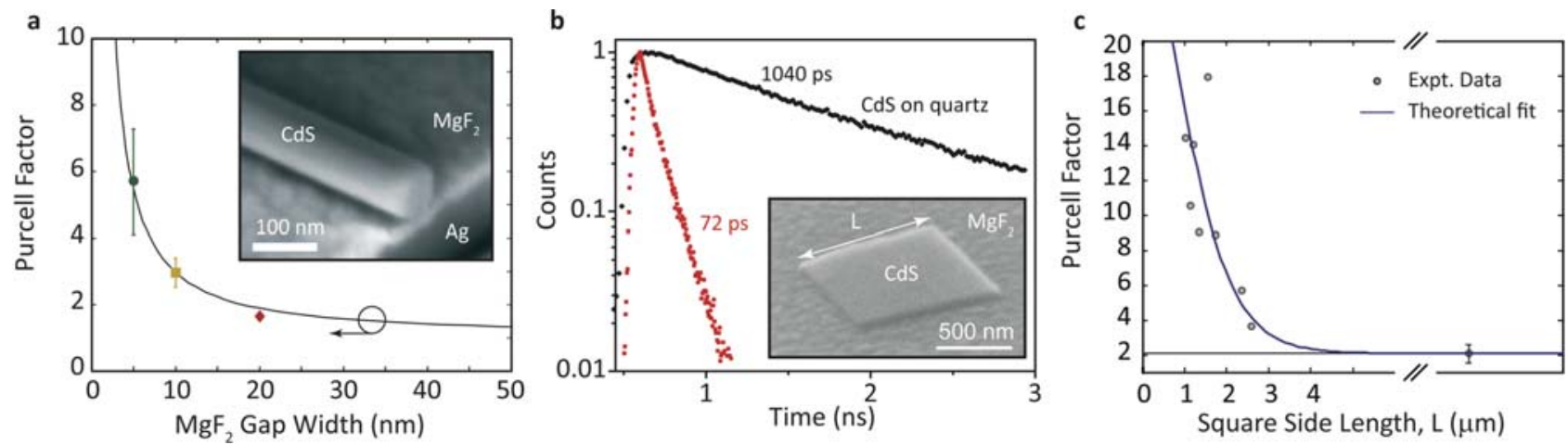

Figure 7 (online color at: www.lpr-journal.org) Observation of the Purcell effect in plasmonic lasers. a) Purcell factors of CdS $I_{2}$ exciton emission from nanowires on a metal surface separated by a thin MgF2 gap layer (see inset), for diameters near optimal confinement $(d=120 \mathrm{~nm} \pm 20 \mathrm{~nm})$. The average Purcell factor for devices with $5 \mathrm{~nm}$ gaps is more than 6 , which is considered to be high for a broad band effect (i.e., without cavity enhancement). b) Time resolved spontaneous emission of a related laser devices consisting of thin CdS squares placed on metal with a similar $\mathrm{MgF}_{2}$ gap layer (see inset) shows a dramatic reduction in lifetime compared with CdS placed on quartz. The combination of both high cavity quality and strong confinement enhances the spontaneous emission rate by 14 times. c) shows the increase in the Purcell effect with the decrease of the square cavity side length (inset of panel b) as a result of the mode volume reduction accompanied by an increasing cavity quality factor. Very large cavities do not benefit from cavity feedback and exhibit a Purcell effect of about 2 due to confinement along the $z$-direction alone. This agrees well with a simple theoretical model taking into account the numerous emission processes. From [9] and [11].

confinement and total internal reflection feedback, which could not be observed in any photon-only control samples consisting of CdS squares on quartz substrates. By controlling the structural geometry we have reduced the number of cavity modes to achieve single mode lasing with a line width about $1.1 \mathrm{~nm}$ (Fig. 6d). High Purcell factors, as large as 20 , were also observed in these laser devices indicating the combination of both strong confinement and feedback (Fig. 7b,c).

Later, Flynnet al. reported the other room temperature operated plasmon laser with emission peak around $1.46 \mu \mathrm{m}$ [12]. They sandwiched a gold-film plasmonic waveguide between optically pumped InGaAs quantum-well gain media and used the two ends of the materials as Fabry-Perot mirrors. The formed Fabry-Perot cavity is with a length of $1 \mathrm{~mm}$ and width of $100 \mu \mathrm{m}$, much larger than the surface plasmon propagation length.

\subsubsection{Photon-based metallic nano-lasers}

The prospect of coherent light sources with footprints comparable to electronics has led to a number of recent works on metal based lasers which utilize metals towards increasing the modal confinement [7,63-68]. While these studies do not examine the laser action of plasmonic modes, they have developed the technology of small lasers by addressing key problems such as heat sinking and electrical contacting. Hill and co-workers demonstrated the first nano-scale electrically driven metal coated laser [63]. Recent work has seen electrically driven devices even under continuous wave operation at room temperature [66]. However, these devices cannot be scaled beyond the diffraction limit. The advantages of such lasers are primarily found in the balance between miniaturizing a laser without inferring significant optical losses by the metal. On the other hand, the metal alone provides a noteworthy advantage as it serves as an integrated electrical contact and heat sink at the same time.

\section{Characteristics of plasmon laser action}

The general trend to miniaturize lasers in past decades has led to clearly observable differences in their operational characteristics compared to conventional devices [69-80]. In particular, small lasers exhibit enhanced spontaneous emission, which modifies threshold behavior and increases noise, and naturally suffer loss of beam directionality when their size approaches the diffraction limit. Due to their extremely small physical size, plasmonic lasers naturally exhibit all of these characteristics to a much greater extent; however, new features are also evident. This includes strong dispersion due to the high gain necessary to attain laser action and the optical response time of plasmon lasers due to both high gain and fast spontaneous emission. This can accentuate more subtle effects such as gain guiding, frequency pulling and gain switching.

In this section, we discuss the consequences of enhanced spontaneous emission and strong dispersion in the operation of plasmon lasers. In particular we focus on Purcell effect, speed, threshold and dispersion. The modification of the threshold behavior and the high losses of metals at optical frequencies are unavoidable. As the dimensions of a laser cavity approach the operating wavelength, the rate of spontaneous emission from excited state to an optical mode can either be enhanced $[9,11,20,81,82]$ or inhibited [83-86], an effect first pointed out by Purcell [87]. This can affect a number of processes within a laser, but most importantly, it causes a drastic spatial redistribution of spontaneous emission $[81,82]$. That is to say, light can be inhibited into some 
modes and preferentially coupled to the laser mode. This can significantly reduce the pump conditions for the onset of laser action and is therefore key to the operation of plasmon lasers. Since the electron collision time of metals is of the order of 100 femtoseconds, plasmon lasers require high gain to operate, which leads to strong gain dispersion and fast response times.

\subsection{Purcell effect}

The Purcell effect is commonly associated with Fermi's Golden rule, which quantifies the rate at which weakly coupled systems can exchange energy with an optical mode. The Purcell factor, $F$, is the emission rate enhancement into all possible modes of an optical structure relative to the emission rate in the absence of that structure. Strong field confinement and high quality factor of the cavity will give a high Purcell factor. There are many examples of spontaneous emission rate enhancement that have been observed in plasmonic systems $[9,11,17-19,88]$. In this review, we focus on those systems that also exhibit laser action. The first example is the hybrid plasmonic nanowire lasers, where the enhancement is due to the plasmon confinement along (Fig. 7a) [9]. The second example is CdS square plasmon laser, where the enhancement is due to the plasmon confinement along and the cavity quality factor Fig. 7b [11]. While these geometries exhibit the most intense spontaneous emission modification in their gap regions where the field is most intense [88], enhancements are also observable to a lesser extent in the CdS gain medium because the confinement is very strong. In both examples, the emission rate was measured by time correlated single photon counting, by weak optical excitation from $100 \mathrm{fs}$ pulses centered at a wavelength of $405 \mathrm{~nm}[9,11]$. In the first case, emission was collected from the sample at $10 \mathrm{~K}$, where the signal was dominated by $\mathrm{I}_{2}$ excitons at a wavelength of $489 \mathrm{~nm}$ with a natural lifetime of about 400 ps [9]. In the second example, emission was collected from the sample at room temperature, where the band edge emission near $506 \mathrm{~nm}$ has a lifetime of approximately $1 \mathrm{~ns}$ [11].

Plasmonic nanowire lasers show a strong increase of the spontaneous emission rate when the gap size between the nanowire and metal surface is decreased, as shown in Fig. 7a. Lifetime measurements reveal a Purcell factor of more than six for a gap width of $5 \mathrm{~nm}$ and nanowire diameters near $120 \mathrm{~nm}$, where hybrid plasmonic modes are most strongly localized. This enhancement factor corresponds to a mode that is a hundred times smaller than the diffraction limit, which agrees well with our mode size calculations, based on the Purcell factor for 1D plasmon waves [9].

A higher Purcell effect is observed in the square laser devices operating at room temperature [11]. Surface plasmons undergo total internal reflection at the device boundaries forming an effective cavity with strong feedback. Figure $7 b$ shows a time correlation histograms for a laser device exhibiting a 14 fold increase in the spontaneous emission rate compared to the natural rate of CdS band edge transitions. It is the combination of both relatively high cavity quality factors $(Q)$ for a plasmonic cavity and strong mode confinement in only one dimension that leads to Purcell factors as large as 18 in the smaller devices. The electric-field intensities in the gap regions of this type of device are at least five times stronger than in the CdS, Purcell factors as high as 100 are anticipated for light-matter interactions within the gap.

The extent of spontaneous emission control in plasmonic nanostructures can be extraordinarily large across a broad range of frequencies. For example, consider the Purcell enhancement factor emitters in close proximity to metaldielectric systems exhibiting mode confinement in one, two, and three dimensions [20], shown in Fig. 8. A planar metaldielectric interface and a thin metal nanowire represent plasmonic systems with one- (1D) and two- (2D) dimensional confinement, respectively, where collective electron oscillations are bound to the surface or wire but are free

a

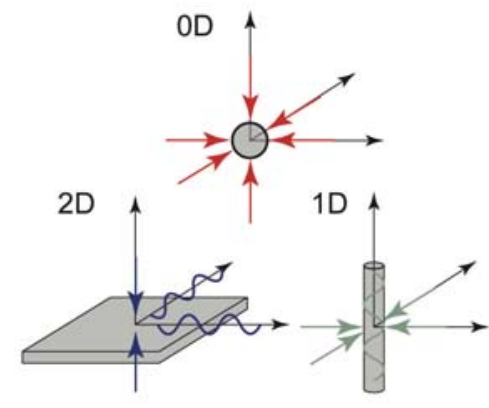

b

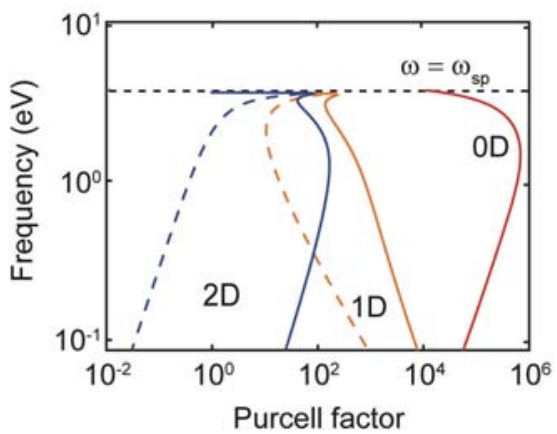

Figure 8 (online color at: www.lpr-journal.org) a) Metal nanostructures supporting surface plasmon modes with varying degrees of confinement. The 1D system is a planar silver/air interface supporting SPPs confined in one dimension. The 2D system is a $40 \mathrm{~nm}$ diameter silver nanowire in air and supports the symmetric zero-order SPP confinement in two dimensions. The 3D system corresponds to a nanosize silver particle confined in all three dimensions. b) Spontaneous-emission-rate enhancement for emitters coupled to SPs with confinement in a varying number of dimensions. The responses for the 1D- and 2D-confined SPPs are divided into intrinsic (broken lines) and cavity-enhanced (solid lines) enhancement factors, where feedback has been introduced through cavity mirrors in a Fabry-Perot configuration. The red solid line shows the limits of plasmonics where nonlocal effects become significant. This limit could be reached with metallic nanoparticle SP resonators. From [20]. 
to propagate in the remaining unbound directions as SPPs. These systems are non-resonant and support SPPs at energies below the plasma frequency. On the other hand, a metal nanoparticle supports a resonant localized SP confined in all three dimensions.

The intrinsic characteristics of SPs alone (i.e. without feedback) can lead to dramatic enhancements in the spontaneous emission rates (Purcell effect) of emitters near metallic nanostructure as shown by the broken lines in Fig. 8. While surface plasmons of a metal dielectric interface mainly experience confinement near the surface plasmon frequency, surface plasmons of metal nanowires achieve significantly higher Purcell factors at shorter frequencies due to an anomalous scaling of the confinement with $\omega^{-2}$ [20]. Anomalous scaling of confinement with frequency may also be found in other types of metallic nanostructures, e. g., two parallel metal planes separated by a dielectric gap exhibit a confinement factor that scales with $\omega^{-1}$ [20].

When feedback is introduced, as in the case of laser devices, the emission rates are further enhanced by a factor proportional to the cavity finesse. In both $1 \mathrm{D}$ - and 2D-confined systems, additional confinement is also possible near the surface-plasmon frequency, where the increased phase index of the SPPs allows a reduction in the cavity length. The solid lines in Fig. 8 show the emission-rate enhancement of 1D and 2D systems with single mode Farby-Perot type cavities. Assuming sufficient feedback to ensure that cavity $Q$ factors are limited by the intrinsic dissipation, leads to significantly higher Purcell factors, even near the surface plasmon frequency where losses are highest.

The smallest possible surface plasmon cavities, such as nanoparticles, provide confinement in all three spatial dimensions. Rather than to specify the maximum Purcell effect for such geometries, Fig. 8 shows the attainable Purcell effect in nanoparticles before nonlocal effects start to become important [20]. At this point, plasmonics losses are expected to increase drastically and the potential for useful enhancements is broadly unexplored. Nevertheless, at this point, it is possible to achieve sub-picosecond spontaneous emission lifetimes with semiconductor materials [89].

\subsection{Modulation speed}

In order to gain a basic theoretical understanding of the characteristics of small lasers, we start with a simple steady state rate equation picture of the photon number of a single laser mode, $s$, and excited electronic state population, $n$, within a small single mode laser device under continuous pumping, $p$. The form of these equations is reminiscent of much of the work from the 1990's on microcavity lasers [90-93]. Particular attention should be paid to the fact that both spontaneous and stimulated processes are proportional to the spontaneous emission rate, $A$, and are thus both modified by the optical environment through the Purcell effect, where the Purcell Factor, $F=A / A_{0}$ where $A_{0}$ is the natural spontaneous emission of the material and the corresponding modal redistribution of emission. A faster spontaneous emission rate into the laser mode, $A_{i}$, relative to the total spontaneous emission rate into all modes, $A$, increases the probability of light coupling into that mode, quantified by the spontaneous emission factor, $\beta=A_{i} / A$. The rate equations are:

$$
\begin{aligned}
& \frac{d n}{d t}=p-A n-\beta \Gamma A s\left(n-n_{0}\right), \\
& \frac{d s}{d t}=\beta A n+\beta \Gamma A s\left(n-n_{0}\right)-\gamma s .
\end{aligned}
$$

Here $p$ is a pump rate, $\Gamma$ is the overlap factor quantifying the spatial distribution of gain relative to the laser mode and $\gamma$ is the total cavity mode loss rate. For a semiconductor laser with a linear gain model we interpret $n_{0}$ as the excited state population at transparency and $\gamma_{g}=\beta \Gamma A s n_{0}$ is the absorption rate due to the gain medium. The rate of change of the carrier density is described by the first equation with the first term accounting for pump rate, the second term for carrier loss due to spontaneous emission and non radiative recombination, and the last term for carrier loss due to stimulated emission. The second equation describes the rate of change of the photon density with the first term accounting for photons generated by spontaneous emission and coupled into the lasing mode, the second term for photons generated by stimulated emission, and the final term for photon loss from the cavity. Note that we have assumed a non-depleting pump rate, $p$, and rapid decay from an upper level to fill the upper laser transition; it is straightforward to apply a similar analysis to systems where a finite number density of carriers in the gain region depletes the pump. Through small signal analysis of the rate equations, the $3 \mathrm{~dB}$ bandwidth for high loss SP cavities can be described as: [20]

$$
f_{3 \mathrm{~dB}}=\frac{\gamma}{\pi} \Omega\left(\omega_{r} / 2 \gamma\right)
$$

where $\Omega(\zeta)=\zeta\left(1-2 \zeta^{2}+2 \sqrt{1+\zeta^{2}\left(\zeta^{2}-1\right)}\right)^{1 / 2}, \omega_{r}=$ $\sqrt{A \gamma r}, r=p_{0} / p_{t h}$, and the plasmon laser's response rate is in the range of $\omega_{r}<2 \pi f_{3 \mathrm{~dB}} / \sqrt{3}<\gamma_{c}$. Through the formula, we can see that the modulation bandwidth of a plasmon laser can be significantly enhanced due to its enhanced spontaneous emission rate (Purcell factor) and short photon lifetime of the nanometer-sized plasmon cavity. The estimated modulation bandwidth for the plasmon laser is in the $\mathrm{THz}$ range (Fig. 9).

\subsection{Lasing threshold behaviours}

From the rate equations in steady state, the emitted laser mode photon number, $s$, and excited state population, $n$, are related by, $p=(1-\beta) A n+\gamma$, and the photon number is described by the quadratic equation,

$$
\gamma s^{2}-s\left[p-p_{t h}^{(n)}\right]-[p / \Gamma]=0
$$




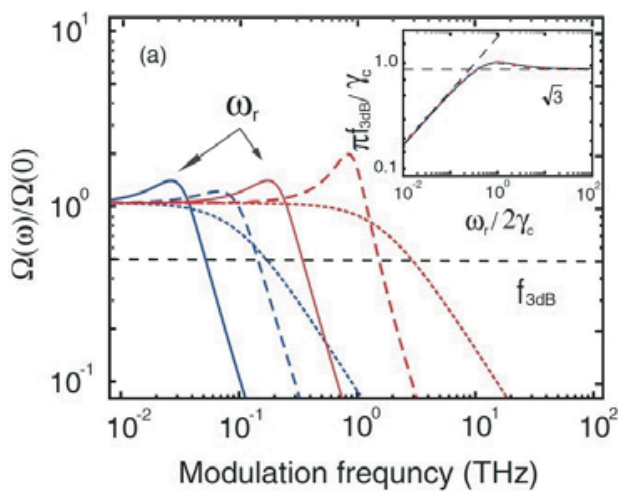

Figure 9 (online color at: www.lpr-journal.org) Small signal modulation response of plasmon lasers. The response functions for 1D (red curves) and 2D (blue curves) plasmon laser cavities $\left(m=1, R_{c}=99.9 \%\right)$ for ideal four level emitters at telecoms frequency $(\omega=0.83 \mathrm{eV})$. Three different pump rates: $r=10$ (solid lines), $r=200$ (dashed lines) and $r \rightarrow \infty$ (dotted line). The $3 \mathrm{~dB}$ modulation bandwidth follows a universal function (insert). At low pump rates, the bandwidth is limited by relaxation oscillations, $f_{3 \mathrm{~dB}}=\sqrt{3} \omega_{r} / 2 \pi$, saturating at $f_{3 \mathrm{~dB}}=\sqrt{3} \gamma_{s p}^{c} / 2 \pi$ for high pump rates due to cavity damping. From [20].

where, $p_{\text {th }}^{(n)}=\left(\gamma+(1-\beta) \gamma_{g}\right) / \beta \Gamma$, with solutions of the form,

$$
\begin{aligned}
\gamma_{S}= & \frac{1}{2}\left(p-p_{t h}^{(n)}\right) \\
& +\frac{1}{2}\left(p+p_{t h}^{(n)}\right)\left[1-\frac{4}{p} \frac{(1-\beta) A n_{\infty}}{\left(1+p_{t h}^{(n)} / p\right)^{2}}\right]^{1 / 2} .
\end{aligned}
$$

Where $n_{\infty}=\left(\gamma+\gamma_{g}\right) / \beta \Gamma A$, is the excited state population where the total gain is equal to the total loss. In the limits of very high and very low pump rates, distinct linear responses for the photon number occur,

$$
\begin{aligned}
& s(p \rightarrow \infty)=\frac{p}{\gamma}-\frac{(1-\beta) A n_{\infty}}{\gamma}, \\
& s(p \rightarrow 0)=\frac{p}{\Gamma p_{t h}^{(n)}}
\end{aligned}
$$

and, correspondingly for the excited state population,

$$
\begin{aligned}
& n(p \rightarrow \infty)=n_{\infty}, \\
& n(p \rightarrow 0)=\frac{n_{\infty} p}{p_{t h}^{(n)}} .
\end{aligned}
$$

It is possible to discern the threshold behaviour of lasers from where these linear trends intersect. However, for small lasers with large spontaneous emission factors, the laser threshold takes on a distinct character to that of conventional lasers. When the usual but essentially ad hoc definition of laser threshold is taken as the pump rate, where $s(p \rightarrow \infty)=$ 0 , we find

$$
p_{t h}^{(s)}=\frac{(1-\beta)}{\beta \Gamma}\left(\gamma+\gamma_{g}\right) \text {. }
$$

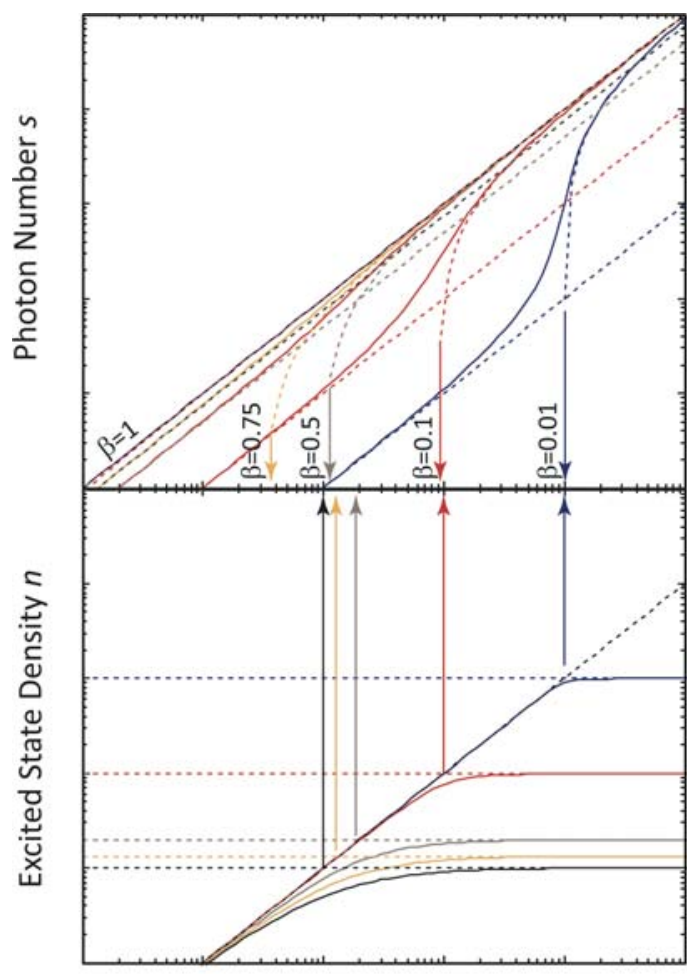

Normalized pump rate $p / \gamma$

Figure 10 (online color at: www.lpr-journal.org) Characteristics of the onset of laser action in small lasers modified by the Purcell Effect. The photon number and excited state population are plotted versus the normalized pump rate. Here we assume conditions that are characteristic of plasmonic lasers, where the cavity loss rate is much larger than the spontaneous emission rate, $A=10^{-4} \gamma$. As mode selectivity increases due to the beta factor, the transition from spontaneous to stimulated emission dominated response becomes smeared.

Another measure of the transition between spontaneous and stimulated emission dominated operation is to monitor the onset of gain clamping, i.e., where $n(p \rightarrow 0)=n_{\infty}$, we find a different expression,

$$
p_{t h}^{(n)}=\frac{1}{\beta \Gamma}\left(\gamma+(1-\beta) \gamma_{g}\right)
$$

As Fig. 10 shows, the point at which laser threshold appears to occur for the light pump curve, does not directly correspond to where the population inversion is clamped. We also note that a conventional definitions can lead to apparently thresholdless laser operation since when $\beta \rightarrow 1$, we find that the kink in the light versus pump curve disappears, as shown in Fig. 10a. However, the term "thresholdless" should be understood to mean "lack of threshold behaviour", as opposed to "an onset of laser action for infinitely small pump rates" [94-105]. Clearly, at $p_{t h}^{(s)}$ the gain has not increased sufficiently, to clamp the population as shown in Fig. 10b.

An alternative, more consistent definition of laser threshold, $p_{t h}^{(\gamma)}$, can be determined from the laser linewidth as a function of pump rate. For large pump powers in excess of 

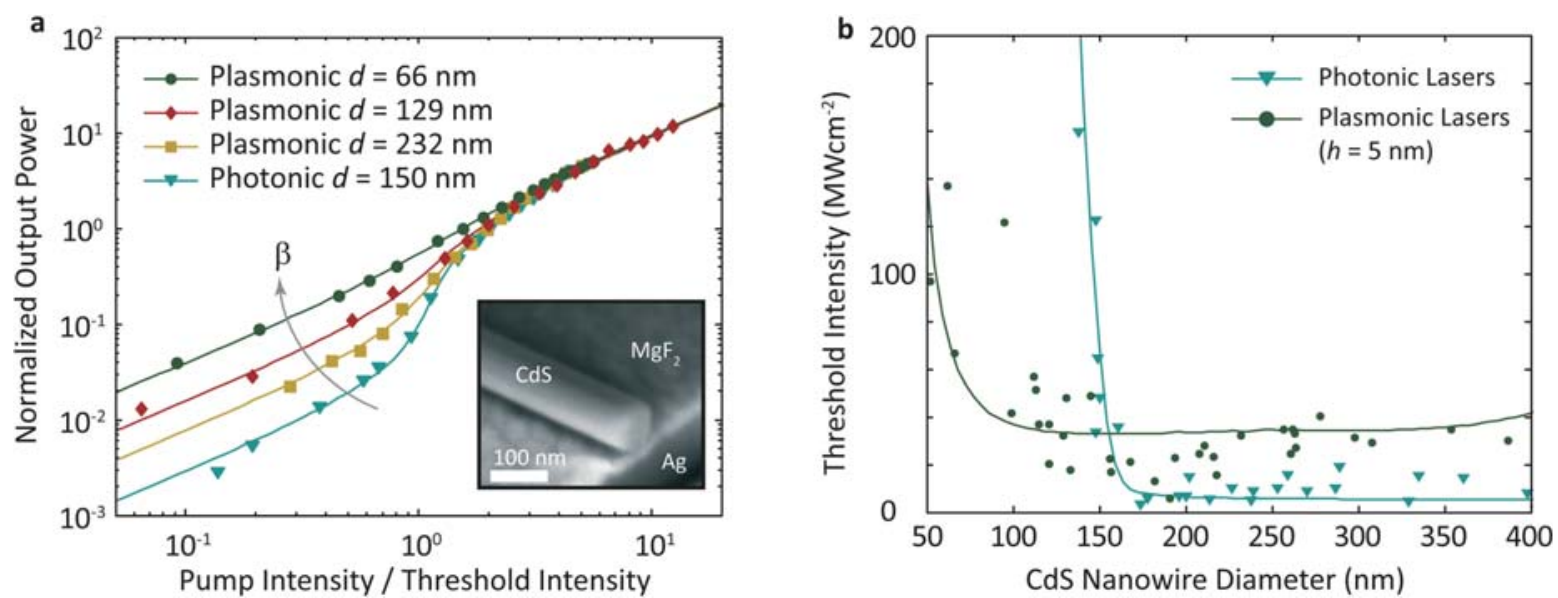

Figure 11 (online color at: www.lpr-journal.org) Modification of conventional laser characteristics by the Purcell Effect in plasmonic nanowire lasers. a) The dependence of measured output power over pump intensity highlights the clear differences in the physics underlying the plasmonic and photonic lasers using a multi-mode lasing model. In particular, our fitting parameter, $x_{0}$, is related to the gain saturation of individual longitudinal laser modes and their lateral mode area. A higher value of $x_{0}$ corresponds to a smaller mode area and a higher $\beta$-factor. Photonic lasers exhibit a clear transition between spontaneous emission and laser operation characterized by $x_{0}=0.026$ for a $150 \mathrm{~nm}$ diameter nanowire. Plasmon lasers, however, show a strong dependence of $x_{0}$ on the nanowire diameter producing much larger values between $x_{0}=0.074$ for larger wires up to $x_{0}=0.380$ for the smallest. b) Threshold intensity of plasmonic and photonic lasers versus nanowire diameter. The experimental data points correspond to the onset of amplified spontaneous emission, where coherent peaks start to appear in the laser spectrum. Amplified spontaneous emission in hybrid plasmonic modes occurs at moderate pump intensities of $20-50 \mathrm{MW} \mathrm{cm}^{-2}$ across a broad range of diameters, while photonic nanowire lasers, with much lower losses, require $5-20 \mathrm{MW} \mathrm{cm}^{-2}$. Note also, that while plasmonic lasers maintain good mode confinement over large range of diameters as shown in e $(50<d<500 \mathrm{~nm})$, photonic lasers exhibit a sharp increase in the threshold for $d<150 \mathrm{~nm}$, due to the loss of confinement within the nanowire and subsequent lack of overlap with the gain region. From [9].

$p_{t h}^{(n)}$ the photon rate equation can be written as,

$$
\frac{d s}{d t}=\beta A n_{\infty}-\gamma^{\prime} s=0
$$

According to this expression, the photon number in the laser mode is fed by spontaneous emission (first term), while light escapes from the cavity at a modified rate $\gamma^{\prime}$ (second term), which accounts for both the loss compensation by stimulated emission and underlying cavity losses. The reader will notice that in steady state, the laser mode loss must still be finite depending on the rate of spontaneous emission. The laser mode's loss rate is given by,

$$
\gamma^{\prime} \approx \frac{\gamma\left(\gamma+\gamma_{g}\right)}{\Gamma p}=\beta \gamma \frac{p_{t h}^{(\gamma)}}{p}
$$

Where we have chosen $p_{t h}^{(\gamma)}=\left(\gamma+\gamma_{g}\right) / \beta \Gamma>p_{t h}^{(n)}>p_{t h}^{(s)}$ While this threshold condition ensures the onset of gain clamping, it does not necessarily imply an increase in laser coherence, since when $\beta \mapsto 1, \gamma^{\prime}=\gamma$ at $p=p_{t h}^{(\gamma)}$.

For lasers with a high $\beta$, there is a clear discrepancy between the apparent onset of laser threshold from the observation of carrier inversion, the light pump curve or the reduction of linewidth since $p_{t h}^{(\gamma)}>p_{t h}^{(n)}>p_{t h}^{(s)}$. This is why plasmonic lasers that clearly exhibit kinks in their light pump response also show much broader linewidths than we might expect. However, all the definitions of threshold here indicate where coherent processes start to dominate. This is when the pump rate is equal to the total cavity loss rate divided by the $\beta$ factor. While conventional lasers have relied on relatively low cavity losses to ensure realistic thresholds, since $\beta \ll 1$, plasmonic lasers can operate at much higher cavity loss rates provided there is a substantial improvement in $\beta$ stemming from a strong Purcell effect and mode selection [90-93]. This behavior has already been seen in plasmonic nanowire lasers [9]. Figure 11 shows normalized light output versus pump curves and laser thresholds for a selection of CdS nanowire lasers on Quartz and Metallic substrates under the same optical pumping conditions. While the plasmonic nanowire lasers have internal cavity losses estimated to be about 10x larger that the photonic nanowire lasers $(\eta \sim 0.1)$, the average plasmonic laser has a threshold no more than a factor of 3 larger than a photonic nanowire laser of the same diameter, as shown in Fig. $11 \mathrm{~b}$. We attribute this to the much larger $\beta$ factors of the plasmonic lasers, as shown in Fig. 11a.

It is quite clear that a more detailed theory is needed to define a laser's threshold, for example using phase space methods [106] or we should change our assertion that the onset of laser action in small devices is a critical phenomenon.

\subsection{Dynamic rate equation analysis}

In this section, we explore the properties of plasmonic lasers pumped with pulses that are short relative to the spontaneous emission lifetime. While extremely fast pump pulses do not 

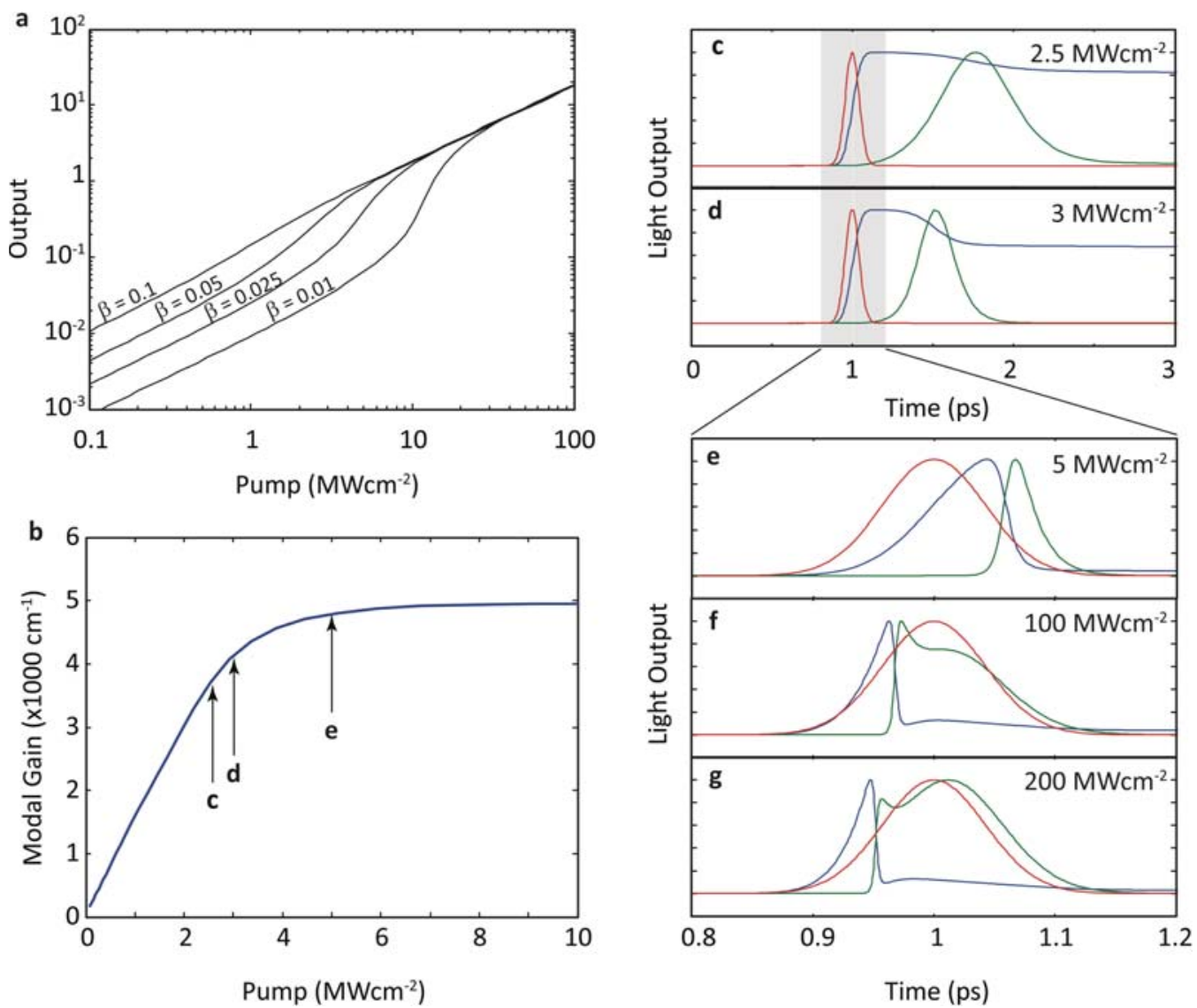

Figure 12 (online color at: www.lpr-journal.org) Light-pump curve and transient response of dynamic rate equation analysis. a) Nonlinear light-pump curve highlights the onset of lasing. We can identify laser action from the dependence on the spontaneous emission factor, which shows the laser's ability to couple directly to the laser mode. b) shows the saturation of the calculated time averaged gain within the laser. The corresponding transient laser responses are shown in c-g). Here, the effect of gain switching can be observed. The red, blue and green curves are the pump, exciton and photon densities respectively. The gain switching leads to a fast output pulse that can be as fast as the pump pulse since the cavity photon lifetime is much shorter than the pump laser pulse. We note that internal dynamics could be limited by the internal dynamics of the gain medium, such as carrier thermalization.

significantly modify the characteristic threshold behaviour of plasmonic lasers, the internal dynamics can be extremely fast due to short cavity lifetime, i.e. high cavity loss, the Purcell effect and large $\beta$ factors. This may be of considerable interest toward new applications area of these laser devices, in particular, high intensity laser physics.

We have solved the rate equations using parameters according to the experimental conditions of the plasmonic nanowire laser work [9]: the incident pump laser pulse, $p(t)=p_{0} \exp \left(-4 \ln 2\left(t-t_{0}\right)^{2} / \Delta t^{2}\right)$ has a full width half maximum pulse width, $\Delta t=100 \mathrm{fs}$; the natural lifetime of bulk CdS I $\mathrm{I}_{2}$ exciton emission is $A_{0}^{-1}=400 \mathrm{ps}$; the Purcell factor is $F=5$, giving a spontaneous emission lifetime of $A^{-1}=80 \mathrm{ps}$; the photon lifetime in the cavity is, $\gamma^{-1}=13$ fs, corresponding to a Fabry-Perot modal cavity loss of $5,000 \mathrm{~cm}^{-1}$, the mode confinement factor, $\Gamma=0.5$; the pump beam has a spot size of $40 \mu \mathrm{m}$; we assume complete absorption of light within the nanowire cross section of $40 \mu \mathrm{m}$ long and $100 \mathrm{~nm}$ diameter; and we have varied the spontaneous emission factor $\beta=[0.01-0.1]$. In the following simplified treatment we have had to set $n_{0}=0$ as the exciton population at transparency is unknown, which would give up to an order of magnitude underestimate of the threshold intensity. Figure 12a shows the output power versus peak pump intensity for the simulated devices. In this case, we can see that the pump intensity necessary to reach threshold is between $1-10 \mathrm{MW} \mathrm{cm}^{-2}$, which is within the expected order of magnitude of what we observed in the plasmonic nanowire laser experiments. When examining the corresponding light-pump curves, one can also immediately see the same effect of the spontaneous emission factor, $\beta$, which smears the transition kink between spontaneous dominated and stimulated dominated emission processes, in a similar manner to the static case.

We have also examined the transient response of the output laser pulse. As expected, this exhibits the traits of gain switching due to the rapid turn on time of these devices, as shown in Fig. 12c-g. In particular, it is important to note that gain switching occurs on the time scale of the plasmon lifetime, which can be as small as $10 \mathrm{fs}$. This suggests that plasmonic lasers could be modulated at extremely high speeds, since relaxation oscillations will have characteris- 
tic frequencies as high as $10 \mathrm{THz}$. Furthermore, Fig. 12c-g reveals the changing shape of the pump pulse for various pump powers above threshold. Just above threshold, a gain switching effect leads to a narrowing laser pulse width. Finally, at very large pump powers, the output photon pulse starts to resemble the shape of the pump pulse. The appearance of transient effects indicates the achievement of laser threshold and this can be corroborated with the saturation of the time averaged gain over the pulse duration (Fig. 12b),

$$
g=\frac{\int \beta \Gamma A s\left(\alpha n-n_{0}\right) d t}{v_{g} \int s d t} .
$$

The fast transient dynamics within these plasmonic lasers could provide the means to deliver energy to the nanoscale on femtosecond timescales, and thus achieve extremely high intensities in the near field. Note that, here we do not account for the carrier thermal relaxation times within their corresponding bands by the interaction with acoustic and/or optical phonons. Recent experiment shows that the luminescence from non-thermalized excitons can be significantly enhanced by the highly concentrated electromagnetic fields supported by plasmonic nanocavities. [89]

\subsection{Radiative and non-radiative loss channels}

In contrast to conventional lasers where external cavities are needed to create feedback, when scaling down the plasmon laser cavity size to deep sub-diffraction region, the surface plasmon resonance will inherently supply the feedback. In this case, the devices deliver energy to the nanoscale plasmonic mode but just release only a small part of their optical energy to the far field before it is dissipated in the metal. A good estimate of the radiative loss of a cavity much smaller than the resonant wavelength, such as a metallic nanoparticle, is the Wheeler-Chu limit [60]: the radiative lifetime, $\tau=3(\lambda / 2 \pi a)^{3} \lambda / c$, where $a$ is the particle radius and $\lambda$ is the resonant wavelength. For such a small metal particle, for instance, that shown in [7], the absorption lifetime is about $25 \mathrm{fs}$ (assuming a quality factor of 15 at $\lambda=532 \mathrm{~nm}$ ), while the radiative lifetime is $8 \mathrm{ps}$; only $0.3 \%$ of generated surface plasmons couple out into the far field. The majority of the energy is coupled to the near field where it is lost as heat. Light leaking into the far field from such devices is just a small fraction of the total energy required to operate them and is therefore of little use. While the light leaking to the far field can be enhanced by lowering the radiation $Q$ i.e. using a bigger metal nanoparticle, it is the light generated in the near field that is of practical use and future applications of these lasers must seek to exploit this. Furthermore, in stark contrast to the highly directional beams of conventional lasers, radiation coupled to the far-field of a localized plasmon laser has a dipole like distribution, which further exacerbates the problem of light collection. These characteristics emphasize the challenges of how we measure and utilize emission from plasmonic lasers as we seek to further reduce their size far beyond the diffraction limit.

\subsection{Giant frequency pulling}

The very high material gain needed to compensate for the losses of metal generates extremely strong cavity mode dispersion [107]. This behavior has been reported in a number of plasmonic lasers with Fabry-Perot type cavities with single transverse laser modes [7,9], as illustrated in Fig. 13. The longitudinal mode spacing, $\Delta \lambda$, is governed by the expression, $\Delta \lambda=\lambda^{2} / 2 n_{g} L$, where $L$ is the wavelength and $n_{g}$ is the effective laser mode group index. For plasmonic nanowire
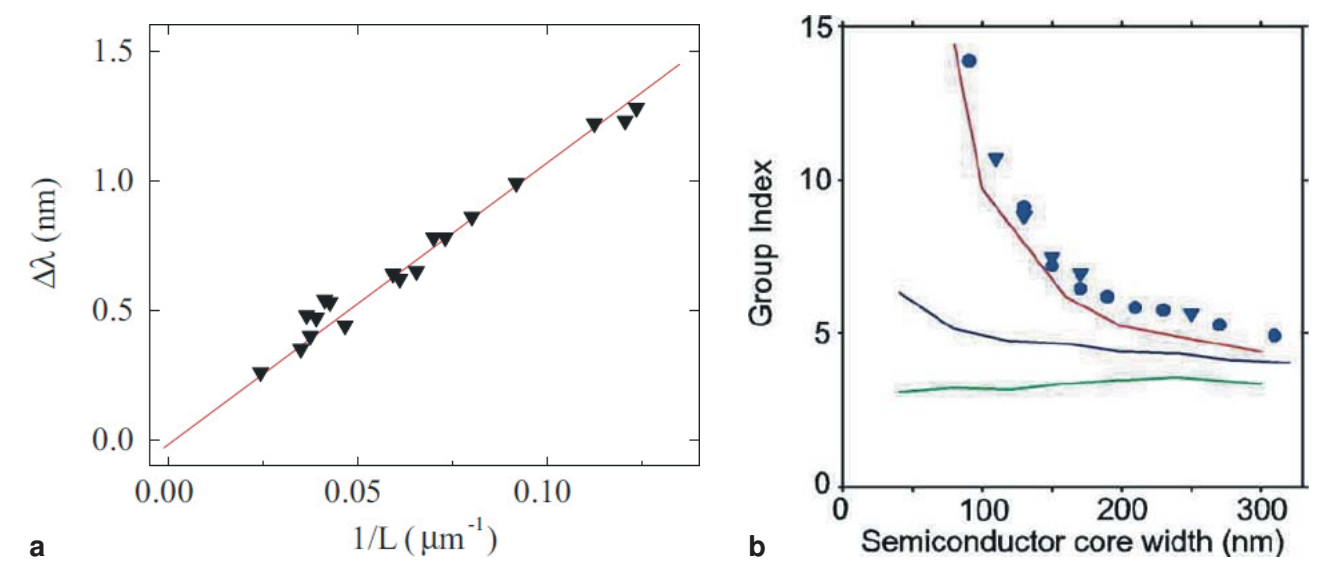

Figure 13 (online color at: www.lpr-journal.org) Observations of large group indexes in plasmonic lasers. a) Longitudinal mode spacing versus inverse nanowire length. The data confirms the Fabry-Pérot like cavity behavior for the plasmonic laser. The red line is a linear fit to the data giving an effective index of 11 [9]. b) Group index, estimated from mode spacing in laser spectra, versus semiconductor core width for metal-insulator-semiconductor-insulator-metal (MISIM) plasmon lasers [7]. Blue circles are estimates from 6 micron long devices and blue triangles are estimates from 3 micron long devices. All measurements at $78 \mathrm{~K}$ except for the two smallest, which were at $10 \mathrm{~K}$. Blue, green and red curves are simulated group indexes: blue for semiconductor only filled MISIM structures (no insulator); green when including the insulator layers; and red when including SiN layers and varying dispersion for the InGaAs core. InGaAs dispersion was varied from $d \varepsilon / d \omega=2 \times 10^{-13} \mathrm{~s}$ for the thinnest device to $d \varepsilon / d \omega=2 \times 10^{-14} \mathrm{~s}$ for the thickest. 

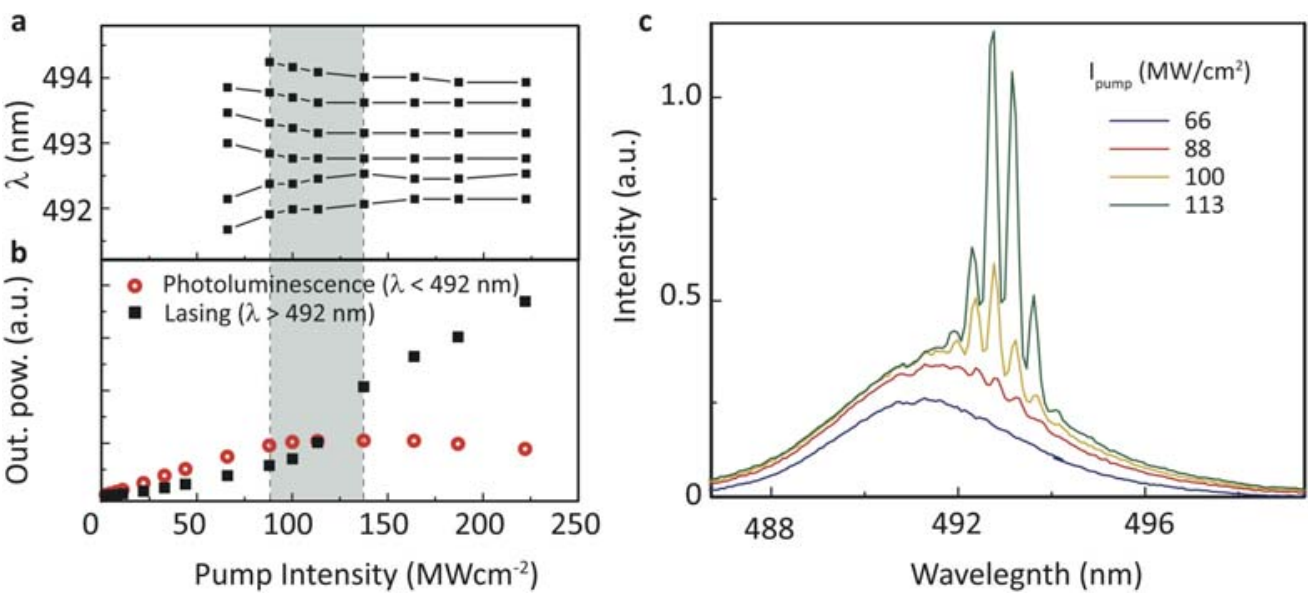

Figure 14 (online color at: www.lpr-journal.org) Frequency pulling in a plasmonic nanowire laser with diameter, $d=112 \mathrm{~nm}$, and length, $L=33.7 \mu \mathrm{m}$. The shaded area in a) and b) indicates the blurred transition from amplified spontaneous emission to lasing, characteristic of small lasers. a) Positions of Fabry-Perot resonances against the peak pump intensity extracted from the spectra in c). For lower pump intensity, the effect of frequency-pulling is clearly seen. At the threshold (on-set of lasing), the frequency pulling becomes constant, indicating that the gain is clamped. b) shows evidence of gain saturation associated with such gain clamping. Here for wavelengths less than $492 \mathrm{~nm}$, only photoluminescence occurs, while lasing occurs for wavelengths greater than $492 \mathrm{~nm}$. While the photoluminescence clearly saturates, the power in the Fabry-Pérot modes continues to increase. c) Emission spectra of the studied plasmonic nanowire laser exhibiting clear Fabry-Pérot peaks.

lasers [9], the mode spacing of all measured devices above threshold followed an approximately linear relationship of $\Delta \lambda$ vs. $1 / L$, confirming that the single transverse mode Fabry-Pérot cavity responses all exhibited essentially the same internal gain (red line in Fig. 13a). The corresponding average group index for theses plasmonic nanowire lasers is about 11, much higher than expected for passive waveguides. For example, photonic nanowire lasers (without metal substrate) have a group index of around 3-4 [108]. Similarly, Martin Hill et al. have observed high mode group indexes in MIM structures [7]. In that work, the authors report a group index that increases with decreasing semiconductor core width, shown in Fig. 13b. Since the core width of these devices strongly affects laser cavity mode propagation loss, it is highly likely that this observation also reflects the high internal gain, necessary to make these lasers operate. The high group index for plasmonic nanowire lasers has been attributed to the strong frequency pulling effect, arising from the large amount of gain required to achieve lasing in the lossy plasmonic system. The nanowire is consequently highly dispersive across the narrow gain spectrum.

In Fig. 14 we show the results of resolving the spectral and light output properties of a plasmonic nanowire laser as a function of the pump power [9]. Amplified spontaneous emission is deemed to start when Fabry-Perot ripples are discernable, in this case near a pump intensity of $88 \mathrm{MW} \mathrm{cm}^{-2}$. The laser threshold is associated with clamping of the internal gain, known as "gain saturation". We therefore expect the frequency pulling effect to "clamp" at the threshold as the gain saturates. At the same time, the spontaneous emission saturates as the stimulated process starts to dominate At this point, laser emission is expected to further increase linearly with the pump intensity. In Fig. $14 \mathrm{~b}$ we can see the increase in frequency pulling throughout the amplified spontaneous emission regime and subsequently, a constant frequency pulled response once lasing has been achieved. At the same time, by comparing those parts of the spectrum that show spontaneous emission, with those that show laser lines, we can see that the underlying photoluminescence saturates, in this case somewhere between $100 \mathrm{MW} \mathrm{cm}^{-2}$ and $113 \mathrm{MW} \mathrm{cm}^{-2}$.

\subsection{Monitoring gain}

We saw from the large frequency pulling effect in Fig. 14 that the gain in plasmonic laser systems can be extremely strong. It is particularly interesting to explore this relationship further as it provides an additional means to study the onset of laser action despite the blurring of the laser threshold. In particular, we can identify that coherence rapidly appears in the emission spectrum of a plasmonic laser, well before the gain is fully clamped. This suggests that the concept of threshold of small lasers is no longer appropriate, since the onset of laser action is no longer a critical phenomenon.

The frequency pulling effect allows us to estimate the material gain of lasers simply by measuring the Fabry-Pérot mode spacing. In conventional lasers this is quite difficult to do as the effect is extremely small; however, in plasmonic lasers the effect is quite pronounced, as illustrated by Fig. 14. The process of estimating the material gain starts from evaluating the average group index, which is related to the nanowire length, $L$, and cavity mode spacing, $\Delta \lambda$, by $n_{g}=\lambda^{2} / 2 L \Delta \lambda$. Figure 15 shows the group index extracted from the peak positions in Fig. 14c. To extract the material gain, we have used a simplified description of the gain from $\mathrm{I}_{2}$ excitons in bulk CdS, which dominate the emission over 

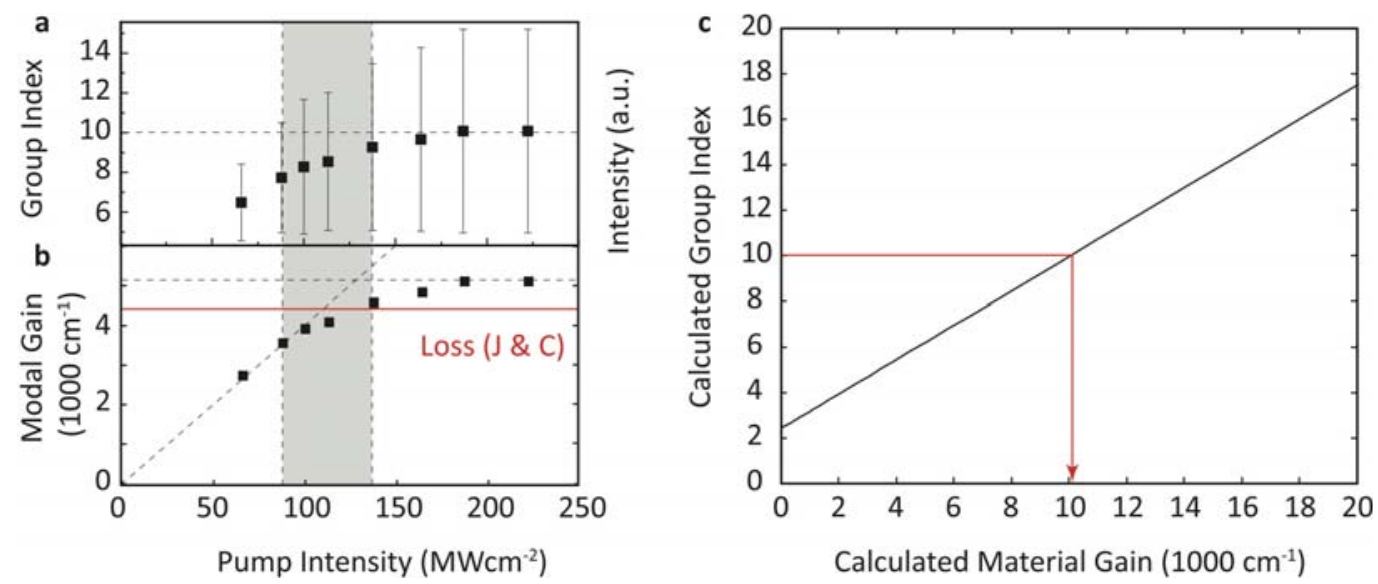

Figure 15 (online color at: www.lpr-journal.org) Estimating material gain from the frequency pulling effect for a plasmonic nanowire laser with a diameter, $d=112 \mathrm{~nm}$, and length, $L=33.7 \mu \mathrm{m}$. The shaded area in a) and b) indicates the transition from amplified spontaneous emission to lasing. a) The average group index calculated from the average mode spacing and the nanowire length (See Fig 14). b) The high group index arises from strong dispersion as a result of the gain and can be used to estimate the internal material gain. In this case, the material gain clamps at about $10,000 \mathrm{~cm}^{-1}$, which concurs well with the estimated modal losses of this plasmonic cavity. c) Calculated relationship between the modal group index and the material gain.

a wide range of pump intensities. The CdS permittivity is described by,

$$
\varepsilon_{\mathrm{CdS}}(E)=\varepsilon_{\mathrm{CdS}}^{\infty}+\frac{2 E_{\mathrm{CdS}} \chi}{E^{2}-E_{\mathrm{CdS}}^{2}-2 i \gamma_{\mathrm{CdS}} E}
$$

Where $E_{\mathrm{CdS}}=2.53 \mathrm{eV}$ and $\gamma_{\mathrm{CdS}}=0.013 \mathrm{eV}$ is the $\mathrm{CdS} \mathrm{I}_{2}$ exciton energy and linewidth respectively and $\varepsilon_{\mathrm{CdS}}^{\infty}=5$ is the background permittivity of CdS. The group index, $n_{g}$, of the mode is then calculated using a finite element method mode solver with a nanowire permittivity, $\varepsilon_{\mathrm{CdS}}(E)$, where the population inversion and gain is parameterized by $\chi$. The relationship between the group index and peak material gain, $g_{\mathrm{CdS}}$, in the CdS nanowire is shown in Fig. 15. The modal gain, $g=\Gamma g_{\mathrm{CdS}}$, within this particular plasmonic nanowire laser is in reasonable agreement with the expected modal losses, $\alpha$,

$$
\alpha=\frac{1}{L_{m}}-\frac{1}{L} \ln R
$$

Where $R \approx 20 \%$ is the reflectivity of the nanowire facets, $500 \mathrm{~cm}^{-1}$ is the cavity loss and $L_{m}^{-1}=4,100 \mathrm{~cm}^{-1}$ is the propagation loss of the plasmonic mode, based on the data of Johnson and Christy [109]. While the material gain, estimated from the frequency pulling experiment, reaches a maximum of about $10,000 \mathrm{~cm}^{-1}$, the actual modal gain is closer to $5,000 \mathrm{~cm}^{-1}$ for an estimated gain-mode overlap factor of $\Gamma=50 \%$ as outlined in the supplementray information of [9]. This matches quite closely with the theoretical losses of $\alpha \approx 4,600 \mathrm{~cm}^{-1}$, from the losses of Silver predicted by Johnson and Christy [109]. However, the quality of the Silver film is likely to play an important role and in the overall losses and this estimate is significantly less than that predicted by the data of Palik [110], which places the theoretical losses at $\alpha>15,000 \mathrm{~cm}^{-1}$.

\subsection{Polarization and diffraction}

In this section we discuss studies of the polarization of scattered light from plasmonic lasers and the diffraction that arises from their small cavities. Such studies have been extremely useful in determining which modes are actually lasing and for unraveling the well-defined mode structure of plasmonic lasers systems.

As an example, we consider again plasmonic nanowire lasers [9], where we have looked in detail at the polarisation properties of scattered light from plasmonic lasers and non-plasmonic (i.e. purely photonic) ones. In the majority of cases, the scattered light polarisation of photonic lasers was perpendicular ( $x$-direction) to the nanowire axis, while for plasmonic lasers, the scattered light polarisation was parallel ( $z$-direction) to the nanowire axis. Figures $16 \mathrm{a}$ and $b$ show results of polarisation angle resolved emission spectra for plasmonic and photonic lasers respectively. This information is critically important as we can identify the internal mode structure of a small laser by its predominant polarization. In this case, a hybrid plasmon mode, whose dominant field components are perpendicular to the metal surface and parallel to the nanowire (Fig. 16c,e), would produce scattered light that is predominantly polarized in $z$-direction. The field distribution is highly localized between the nanowire and metal surface, with a modal area that can be as small as $\lambda^{2} / 400$ (Fig. 16c). Lasers exhibiting polarization along the nanowire axis, therefore lase in highly confined plasmonic modes. For nanowire diameters less than $150 \mathrm{~nm}$, this is the only scattering polarization that is supported by the plasmonic lasers. However, plasmonic lasers with diameters larger than $150 \mathrm{~nm}$ can also support a photonic mode, which is not bound to the metal surface and, in rare cases, leads to lasing that scatters $x$-polarized radiation in the far field. On the other hand, the dominant electric field components of the photonic modes of the nanowire lasers are parallel to the quartz substrate (Fig. 16d and f), 

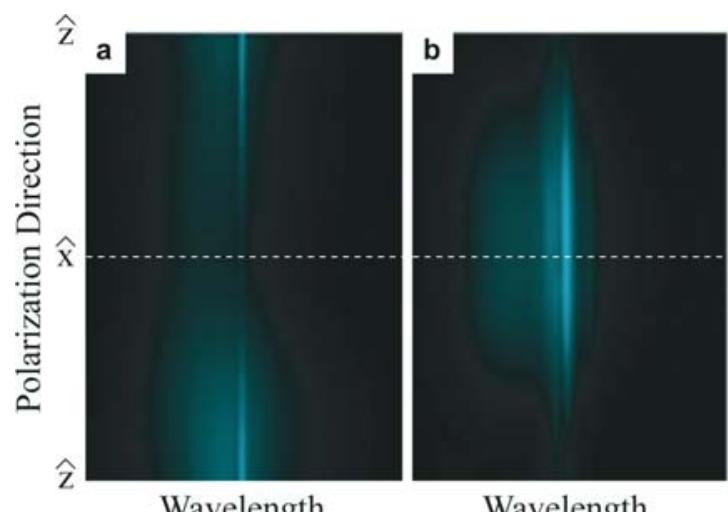

Wavelength
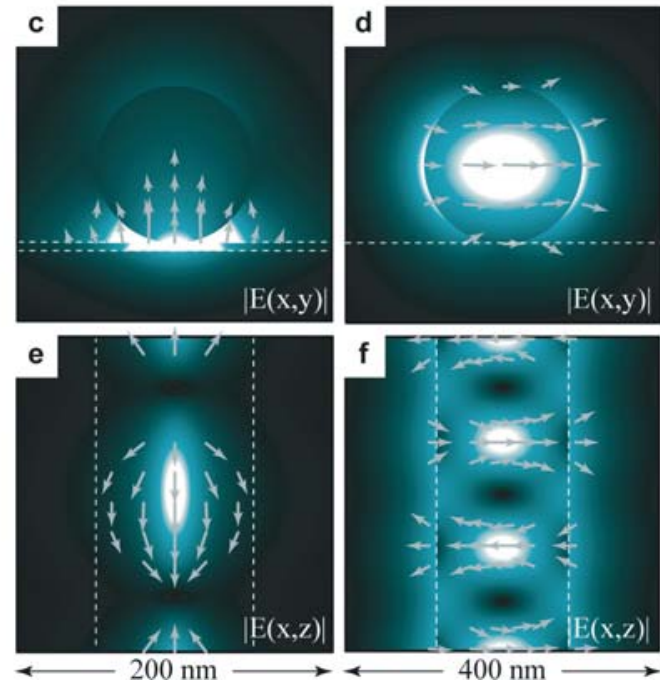

Figure 16 (online color at: www.lpr-journal.org) Nanolaser Polarization dependence. $a$ and $b$ are experimental maps of emission polarisation of scattered light from the nanowire end-facets of a plasmon laser and a control photonic laser, respectively. A dependence on polarisation direction is apparent for both the narrow laser lines as well as the broad background spontaneous emission. While plasmon laser emission is polarised parallel to the nanowire axis, nanowire emission is polarized perpendicular to the nanowire axis. $c-f$ are maps of $|E|$ fields of the plasmonic and photonic lasing modes in the $x / y(\mathrm{c}, \mathrm{d})$ and $x / z(\mathrm{e}, \mathrm{f})$ planes, where the arrows indicate the direction of the $|E|$ field. The $|E|$ field of the plasmon laser mode is predominantly polarised along the nanowire axis and scatters most effectively to radiation with a similar polarisation.

producing scattered light that is predominantly polarized in the $x$-direction. These photonic modes exhibit diffraction limited confinement and are 'cut-off' for diameters smaller than $140 \mathrm{~nm}$, inhibiting laser action.

To date, there have not been any detailed studies examining the extent of diffraction from plasmonic lasers. However, to illustrate the relevance of diffraction, we refer to the recent work on room temperature plasmonic lasers based on semiconductor nano-squares [11]. Figure 17 shows the light collected from a plasmonic laser from the surface normal into and NA $=0.4$ and at an angle of 75 degree to the surface normal into an NA $=0.2$. The same clear

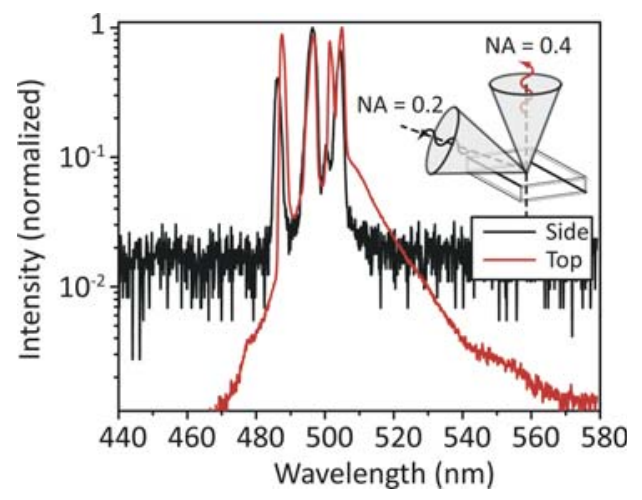

Figure 17 (online color at: www.lpr-journal.org) Emission spectrum above threshold for a multi-mode plasmonic laser for different collection angles. Light collection at large angles from the surface normal (black line) is dominated by the scattering from the lasing cavity modes, while near the surface normal direction a much larger spontaneous emission signal is observable. The modes of the laser diffract significantly after out-coupling.

laser lines are observable over the broad range of angles indicating strong diffraction of the light from the near field. Furthermore, light collected along the surface normal shows a strong spontaneous emission background, while at an angle, the spontaneous emission is strongly suppressed, as one would expect a Lambertian-like profile from spontaneous emission near a metal surface. The reader should refer back to our previous discussion on monitoring laser threshold by the kink in the light pump curve with strongly diffracting laser modes.

\section{Perspective and challenges}

Today, lasers drive scientific and technological innovation in virtually every facet of modern life. With the emerging plasmon laser technology, the question about their unique applications arises. Before we look into areas of potential applications, let us summarize the differentiators of plasmon lasers from conventional lasers, i.e. laser sources that amplify surface plasmons instead of photons. A key advantage of the plasmon laser is its ability to provide amplification of optical modes significantly smaller than the diffraction limit of light. The increased light matter coupling strength in nanoscale plasmon laser cavity induces enhancements of both spontaneous and stimulated emission rates beyond that available to diffraction limited light which enable plasmon lasers with very fast response times. Furthermore, the powerdensity of plasmon laser is much higher due to the strong field confinement. With the ability to generate high intensity electromagnetic radiation at the nanoscale in femtosecond timescales, plasmon lasers can bring fundamentally new capabilities to a variety of fields. Here, as examples, we briefly discuss their potential applications in photonic integrated circuits, sensors \& bio-medical applications and ultra-fast spectroscopy.

Soon after the introduction of electrical circuit integration (ICs) in the 1950's and 60's, a similar scheme 
of photonic circuit integration was proposed by Miller in 1969 [111]. Like any exponential scaling trend, it is just a matter of time when photonic circuit functionality and thus the individual device footprint and performance should enter the sub-wavelength regime. However, one of the main bottle-necks for down scaling photonic circuits remains the lack of an integrated and efficient light source on the chip. Nanoscale plasmon lasers could offer a solution for a light source towards ultra-scaled photonic circuits with unprecedented modulation bandwidth. Furthermore, with integration space and footprint becoming more and more tangible not only the small physical size of plasmon lasers in general will become a critical differentiation, but also the synergies between electronics and photonics are becoming key design factors. For instance, the metal employed in plasmon lasers not only confines the optical field, but also can be used as electrodes and heat sinking simultaneously.

Optical metrology and in particular, sensing applications, are becoming extremely important in the bio-medical arena. Current market-size estimations talk about a 45 billion dollar market in the United States alone [112]. Technology development for sensing biological as well as chemical substances for health treatments and/or chemical trace detections is therefore at high demand. The strong electric field confinement in plasmon laser could be utilized to amplify very weak effects. This could be deployed for detecting single molecules, allowing for extremely sensitive bio-detection. With the high photon density and small optical mode size, nanoscale plasmon lasers could also become useful as manipulators and cutting tools of biological tissues, e.g. affected cancer cells, with unprecedented surgical precision for health care treatments.

As established in Sect. 4, plasmonic nanolasers are potentially able to provide ultra-fast (fs-scale or faster) optical processes. Even though the absolute average optical power provided by these tiny lasers is small, the optical intensity can be quite high due to the extremely small mode size. These nanolasers can therefore be envisioned to become a platform for ultra-fast spectroscopy with unprecedented footprint, energy and ultimate cost functions.

While we have outlined some interesting opportunities for plasmon lasers, it is important to also discuss immediate and potential challenges of such novel devices. Addressing such challenges is significant towards highlighting bottlenecks and paving a path for future research. While the outlook to commercialize plasmon lasers looks promising, there are specific caveats that need to be addressed. Firstly, the large momentum mismatch of light inside and outside of a nanometer-sized cavity results in beam diffraction into all directions once the light exits the laser cavity. This inhibits directional emission of the laser light, which is one of the key advantages of a classical laser. Secondly, many realistic applications would require a failure prone and cheap method of pumping the laser. Such requirements would clearly favor electrical pumping methods. Such a task requires innovative designs of device structures and electrical contacts without perturbation of the optical mode. Thirdly, for large scale integration, the assembly of devices into light-source arrays is necessary favoring top-down processing, yet many of the current plasmon laser demonstrations have relied on bottom up semiconductor growth techniques and post growth assembly.

In summary, as history has shown, with the demonstration of the first laser, the exact technological scope and more importantly the sociological impact of such inventions is often difficult to predict and might even take many years from the moment of solving all device challenges to actual implementation of a product. However, with progressing processing technology, device and chip integration skills and the expanding knowledge about the internal processes on a fundamental level, we can expect to achieve rapid advances in plasmon laser technology that are bright and unlimited.

Acknowledgements. This work was supported by a Multidisciplinary University Research Initiative grant (Air Force Office of Scientific Research, FA9550-10-1-0264).

Received: 13 October 2011, Revised: 23 January 2012, Accepted: 31 January 2012

Published online: 27 February 2012

Key words: Surface plasmon, laser, nanophotonics, ultrafast, miniaturization.

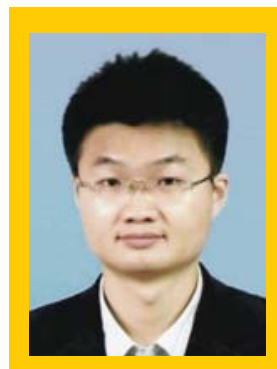

Ren-Min Ma received the Ph. D. degree in Physics from Peking University, China in 2009. His dissertation was focused on semiconductor physics and devices in low dimensional structures and received the National Top $100 \mathrm{Ph}$. D. dissertations of China Award. He is a Postdoctoral Scholar at UC Berkeley since 2009. His current research interests include nanoscale materials, optics, and photonics.

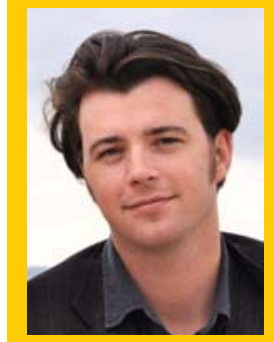

Rupert F. Oulton is a UK Engineering and Physical Sciences Research Council Fellow and Leverhulme Lecturer at Imperial College London, since 2010 . He graduated with a Ph. D. in physics from Imperial College London on the physics of wavelength scale semiconductor optical devices and went onto research plasmonics and metamaterials at UC Berkeley as a Research Associate. His current research interests include the linear and nonlinear optics of metallic nanostructures, metal-based lasers and quantum optics.

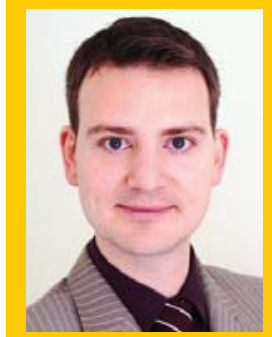

Volker J. Sorger received his Ph. D. and M.S. from the University of California - Berkeley and University of Texas at Austin, respectively, where he conducted research in the fields of nanoscale optoelectronics, optics beyond the diffraction limit of light, nanoelectronics , and novel materials exploration. Currently he is a leading post-doctoral researcher at the NSF Nanoscience and Engineering Center, and a member of 
the DOE funded Energy Frontier Research Center for LightMaterial Interactions in Energy Conversion at UC Berkeley. His research areas include enhanced light-matter-interactions, opto-electronics, silicon photonics, plasmonics, nanotechnology \& materials, photovoltaics and solar-to-energy conversion.

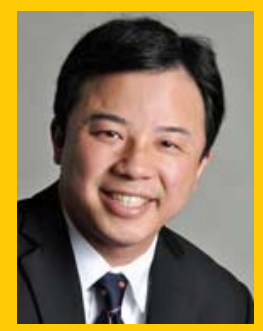

Xiang Zhang is the inaugural Ernest S. Kuh Endowed Chaired Professor at UC Berkeley and the Director of NSF Nanoscale Science and Engineering Center (NSEC). He is also a Faculty Scientist at Lawrence Berkeley National Laboratory (LBNL). Professor Zhang is an elected member of National Academy of Engineering (NAE) and Fellow of four scientific societies: APS (The American Physical Society), OSA (The Optical Society of America), AAAS (The American Association for the Advancement of Science), and SPIE (The International Society of Optical Engineering). His current research focused on nano-scale science and technology, materials physics, photonics and bio-technologies.

\section{References}

[1] T. H. Maiman, Nature 187, 493-494(1960).

[2] NIF's future ignites with 192-beam shot, LLNL community news, https://newsline.1lnl.gov/_rev02/articles/2009/mar/03. 06.09-nif.php, date of last visit: 18 February 2012.

[3] NIF breaks megaJoule barrier, LLNL community news, https://newsline.1lnl.gov/_rev02/articles/2009/mar/03.13. 09-nif.php, date of last visit: 18 February 2012.

[4] J. Klein and J. D. Kafka, Nat. Photonics 4, 289 (2010).

[5] P. B. Corkum and F. Krausz, Nat. Phys. 3, 381-387 (2007).

[6] E. Murphy, Nat. Photonics 4, 287(2010).

[7] M. T. Hill, M. Marell, E. S. P. Leong, B. Smalbrugge, Y. Zhu, M. Sun, P. J. V. Veldhoven, E. J. Geluk, F. Karouta, Y. S. Oei, R. Nötzel, C.-Z. Ning, and M. K. Smit, Opt. Express 17, 11107-11112 (2009).

[8] M. A. Noginov, G. Zhu, A. M. Belgrave, R. Bakker, V. M. Shalaev, E. E. Narimanov, S. Stout, E. Herz, T. Suteewong, and U. Wiesner, Nature 460, 1110-1113 (2009).

[9] R. F. Oulton, V. J. Sorger, T. Zentgraf, R. M. Ma, C. Gladden, L. Dai, G. Bartal, and X. Zhang, Nature 461, 629-632 (2009).

[10] S.-H. Kwon, J.-H. Kang, C. Seassal, S.-K. Kim, P. Regreny, Y.-H. Lee, C. M. Lieber, and H.-G. Park Nano, Lett. 10, 3679-3683 (2010).

[11] R. M. Ma, R. F. Oulton, V. J. Sorger, G. Bartal, and X. Zhang, Nat. Mater. 10, 110-113 (2011).

[12] R. A. Flynn, C. S. Kim, I. Vurgaftman, M. Kim, J. R. Meyer, A. J. Mäkinen, K. Bussmann, L. Cheng, F.-S. Choa, and J. P. Long, Opt. Express 19, 8954 (2011).

[13] M. J. H. Marell, B. Smalbrugge, E. J. Geluk, P. J. V. Veldhoven, B. Barcones, B. Koopmans, R. Nötzel, M. K. Smit, and M. T. Hill, Opt. Express 19, 15109-15118(2011).

[14] A. M. Lakhani, M.-K. Kim, E. K. Lau, and M. C. Wu, Opt. Express 19, 18237-18245 (2011).

[15] C.-Y. Wu, C.-T. Kuo, C.-Y. Wang, C.-L. He, M.-H. Lin, H. Ahn, and S. Gwo, Nano Lett., dx.doi.org/10.1021/ nl2022477 (2011).
[16] M. I. Stockman, J. Opt. 024004 (2010).

[17] T. Liebermann and W. Knoll, Coll. Surf. 171, 115-130 (2000).

[18] P. Anger, P. Bharadwaj, and L. Novotny, Phys. Rev. Lett. 96, 113002 (2006).

[19] A. Kinkhabwala, Z. Yu, S. Fan, Y. Avlasevich, K. Müllen, and W. E. Moerner, Nat. Photonics 3, 654-657 (2009).

[20] D. Genov, R. Oulton, G. Bartal, and X. Zhang, Phys. Rev. B 83, 245312 (2011).

[21] W. Srituravanich, L. Pan, Y. Wang, C. Sun, D. B. Bogy, and X. Zhang, Nat. Nanotechnol. 3, 733-737 (2008).

[22] Y. Kim, S. Kim, H. Jung, E. Lee, and J. W. Hahn, Opt. Express 17, 19476-19485 (2009).

[23] Z. Xie, W. Yu, T. Wang, H. Zhang, Y. Fu, H. Liu, F. Li, Z. Lu, and Q. Sun, Plasmonics 6, 565-580 (2011).

[24] W. A. Challener, C. Peng, A. V. Itagi, D. Karns, W. Peng, Y. Peng, and X. Yang, Nat. Photonics 3, 220-224 (2009).

[25] B. Stipe, T. Strand, C. Poon, and H. Balamane, Nat. Photonics 4, 484-488 (2010).

[26] H. Kano and S. Kawata, Opt. Lett. 21, 1848-1850 (1996).

[27] S. Nie and S. R. Emory, Science 275, 1102-1106 (1997).

[28] K. Kneipp, Y. Wang, H. Kneipp, L. Perelman, I. Itzkan, R. Dasari, and M. Feld, Phys. Rev. Lett. 78, 1667-1670 (1997).

[29] M. Danckwerts and L. Novotny, Phys. Rev. Lett. 98, 026104 (2007).

[30] J. Anker, W. Hall, O. Lyandres, N. Shah, J. Zhao, and R. P. Van Duyne, Nature Mater. 7, 442-453 (2008).

[31] R. T. Hill, J. J. Mock, Y. Urzhumov, D. S. Sebba, S. J. Oldenburg, S.-Y. Chen, A. Lazarides, A. Chilkoti, and D. R. Smith, Nano Lett. 10, 4150-4154 (2010).

[32] A. J. Babadjanyan, N. L. Margaryan, and K. V. Nerkararyan, J. Appl. Phys. 87, 3785-3788 (2000).

[33] M. Stockman, Phys. Rev. Lett. 93, 137404 (2004).

[34] C. Ropers, D. R. Solli, C. P. Schulz, C. Lienau, and T. Elsaesser, Phys. Rev. Lett. 98, 043907(2007).

[35] C. Ropers, C. C. Neacsu, T. Elsaesser, M. Albrecht, M. B. Raschke, and C. Lienau, Nano Lett. 7, 2784 2788(2007).

[36] E. Verhagen, M. Spasenović, A. Polman, and L. Kuipers, Phys. Rev. Lett. 102, 203904 (2009).

[37] H. Choi, D. F. Pile, S. Nam, G. Bartal, and X. Zhang, Opt. Express 17, 7519-7524 (2009).

[38] S. Vedantam, H. Lee, J. Tang, J. Conway, M. Staffaroni, and E. Yablonovitch, Nano Lett. 9, 3447-3452 (2009).

[39] D. J. Bergman and M. I. Stockman, Phys. Rev. Lett. 90, 027402 (2003).

[40] J. Seidel, S. Grafstrom, and L. Eng, Phys. Rev. Lett. 94, 177401 (2005).

[41] M. Ambati, S. H. Nam, E. Ulin-Avila, D. A. Genov, G. Bartal, and X. Zhang, Nano Lett. 8, 3998 (2008).

[42] S. A. Maier, Plasmonics: Fundamentals and Applications (Springer, New York, 2007)

[43] B. Steinberger, A. Hohenau, H. Ditlbacher, A. L. Stepanov, A. Drezet, F. R. Aussenegg, A. Leitner, and J. R. Krenn, Appl. Phys. Lett. 88, 094104 (2006).

[44] C. Reinhardt, S. Passinger, B. N. Chichkov, C. Marquart, I. P. Radko, and S. I. Bozhevolnyi, Opt. Lett. 31, 1307-1309 (2006).

[45] T. Holmgaard and S. I. Bozhevolnyi, Phys. Rev. B 75, 245405 (2007)

[46] E. Verhagen, M. Spasenovic, A. Polman, and L. Kuipers, Phys. Rev. Lett. 102, 203904 (2009). 
[47] S. I. Bozhevolnyi, V. S. Volkov, E. Devaux, and T. W. Ebbesen, Phys. Rev. Lett. 95, 046802 (2005).

[48] D. F. P. Pile, T. Ogawa, D. K. Gramotnev, T. Okamoto, M. Haraguchi, M. Fukui, and S. Matsuo, App. Phys. Lett. 87, 061106 (2005).

[49] J. A. Dionne, H. J. Lezec, and H. A. Atwater, Nano Lett. 6, 1928-1932 (2006).

[50] D. F. P. Pile, D. K. Gramotnev, R. F. Oulton, and X. Zhang, Opt. Express 15, 13669-13674 (2007).

[51] R. Oulton, V. Sorger, D. A. Genov, D. F. P. Pile, and X. Zhang, Nat. Photonics 2, 496-500 (2008).

[52] X.-Y. Zhang, A. Hu, J. Z. Wen, T. Zhang, X.-J. Xue, Y. Zhou, and W. W. Duley, Opt. Express 18, 18945-18959 (2010).

[53] H.-S. Chu, E.-P. Li, and R. Hedge, Appl. Phys. Lett. 96, 221103 (2010).

[54] R. M. Ma, L. Dai, and G. G. Qin, Appl. Phys. Lett. 90, 093109 (2007).

[55] R. M. Ma, L. Dai, H. B. Huo, W. J. Xu, and G. G. Qin, Nano Lett. 7, 3300-3304 (2007).

[56] S. Xiao, V. P. Drachev, A. V. Kildishev, X. Ni, U. K. Chettiar, H.-K. Yuan, and V. M. Shalaev, Nature 466, 735-738(2010).

[57] S. Wuestner, A. Pusch, K. Tsakmakidis, and J. Hamm, Phys. Rev. Lett. 105, 127401 (2010).

[58] M. Stockman, Phys. Rev. Lett. 106, 156802 (2011).

[59] N. I. Zheludev, S.L. Prosvirnin, N. Papasimakis, and V. A. Fedotov, Nat. Photonics 2, 351-354 (2008).

[60] H. A. Wheeler, Proc. IRE 35, 1479-1484 (1947).

[61] R. M. Ma, L. Dai, H. B. Huo, W. Q. Yang, G. G. Qin, P. H. Tan, C. H. Huang, and J. Zheng, Appl. Phys. Lett. 89, 203120 (2006).

[62] R. M. Ma, L. Dai, and G. G. Qin, Nano Lett. 7, 868-873 (2007).

[63] M. T. Hill, Y.-S. Oei, B. Smalbrugge, Y. Zhu, T. de Vries, P. J. van Veldhoven, F. W. M. van Otten, T. J. Eijkemans, J. P. Turkiewicz, H. de Waardt, E. J. Geluk, S.-H. Kwon, Y.-H. Lee, R. Nötzel, and M. K. Smit, Nat. Photonics 1, 589-594 (2007).

[64] R. Perahia, T.P.M. Alegre, A.H. Safavi-Naeini, and O. Painter, Appl. Phys. Lett. 95, 201114 (2009).

[65] K. Yu, A. Lakhani, and M. C. Wu, Opt. Express 18, 87908799 (2010).

[66] C.-Y. Lu, S.-W. Chang, S. L. Chuang, T. D. Germann, and D. Bimberg, Appl. Phys. Lett. 96, 251101 (2010).

[67] M. P. Nezhad, A. Simic, O. Bondarenko, B. Slutsky, A. Mizrahi, L. Feng, V. Lomakin, and Y. Fainman, Nat. Photonics 4, 395-399 (2010).

[68] K. Ding, Z. Liu, L. Yin, H. Wang, R. Liu, M. T. Hill, M. J. H. Marell，P. J. van Veldhoven， R. Nötzel， and C. Z. Ning, Appl. Phys. Lett. 98231108 (2011).

[69] A. F. Levi, S.L. McCall, S. J. Pearton, and R. A. Logan, IEEE Electron.Lett. 29, 1666-1667 (1993).

[70] T. Baba, M. Fujita, A. Sakai, M. Kihara, and R. Watanabe, IEEE Photon. Technol. Lett. 9, 878-880 (1997).

[71] K. Srinivasan, M. Borcelli, O. Painter, A. Stintz, and S. Krishna, Opt. Express 14, 1094-1105 (2006).

[72] J. P. Zhang, D. Y. Chu, S. L. Wu, S. T. Ho, W. G. Bi, C. W. Tu, and R. C. Tiberio, Phys. Rev. Lett. 75, 2678 (1995).

[73] M. H. Huang, S. Mao, H. Feick, H. Yan, Y. Wu, H. Kind, E. Weber, R. Russo, and P. Yang, Science 292, 1897-1899 (2001).

[74] H. Park, S. Kim, S. Kwon, Y. Ju, J. Yang, J. Baek, S. Kim, and Y. H. Lee, Science 305, 1444-1447 (2004).
[75] S. Tomljenovic-Hanic, C. M. Sterke, M. J. Steel, B. J. Eggleton, Y. Tanaka, and S. Noda, Opt. Express 15, 17248-17253 (2007).

[76] A. J. Danner, J. C. Lee, J. J. Raftery Jr., N. Yokouchi, and K. D. Choquette, Electron. Lett. 39, 1323-1324 (2003).

[77] K. Nozaki, H. Watanabe, and T. Baba, Appl. Phys. Lett. 92, 021108 (2008).

[78] H. Cao, J. Phys. A, Math. Gen. 38, 10497-10535 (2005).

[79] H. Altug, D. Englund, and J. Vuckovic, Nat. Phys. 2, 484488 (2006).

[80] B. Ellis, M. A. Mayer, G. Shambat, T. Sarmiento, J. Harris, E. E. Haller, and J. Vuckovic, Nat. Photonics 5, 297-300 (2011).

[81] M. P. van Exter, G. Nienhuis, and J. Woerdman, Phys. Rev. A 54, 3553-3558 (1996).

[82] G. Solomon, M. Pelton, and Y. Yamamoto, Phys. Rev. Lett. 86, 3903-3906 ( 2001).

[83] E. Yablonovitch, Phys. Rev. Lett. 58, 2059 (1987).

[84] D. Englund, D. Fattal, E. Waks, G. Solomon, B. Zhang, T. Nakaoka, Y. Arakawa, Y. Yamamoto, and J. Vuckovic, Phys. Rev. Lett. 95, 013904 (2005).

[85] M. Fujita, S. Takahashi, Y. Tanaka, T. Asano, and S. Noda, Science 308, 1296-1298 (2005).

[86] K. Kounoike, M. Yamaguchi, M. Fujita, T. Asano, J. Nakanishi, and S. Noda, Electron. Lett. 41, 1402-1403 (2005).

[87] E. M. Purcell, Phys. Rev. 69, 681 (1946).

[88] V. J. Sorger, N. Pholchai, E. Cubukcu, R.F. Oulton, P. Kolchin, C. Borschel, M. Gnauck, C. Ronning, and X. Zhang, Nano Lett. 11(11), 4907-4911 (2011).

[89] C.-H. Cho, C. O. Aspetti, M.E. Turk, J. M. Kikkawa, S.W. Nam, and R. Agarwal, Nat. Mater. 10, 669-675 (2011).

[90] L. W. Casperson, J. Appl. Phys. 12, 5194-5201 (1975).

[91] H. Yokoyama and S. Brorson, J. Appl. Phys. 66, 4801-4805 (1989).

[92] G. Bjork and Y. Yamamoto, IEEE J. Quant. Electron. 27, 2386-2396(1991)

[93] H. Yokoyama, K. Nishi, T. Anan, Y. Nambu, S. D. Brorson, E. P. Ippen, and M. Suzuki, Opt. Quant. Electron. 24 S245S272 (1992).

[94] D. Martini and G. R. Jacobovitz, Phys. Rev. Lett. 60, 1711 (1988).

[95] G. P. Agrawal and G. Gray, Appl. Phys. Lett. 59, 399 (1991).

[96] G. Bjork, A. Karlsson, and Y. Yamamoto, Appl. Phys. Lett. 60, 304 (1992).

[97] G. Bjork, A. Karlsson, and Y. Yamamoto, Phys. Rev. A 50, 1675 (1994).

[98] K. An, J. J. Childs, R. R. Dasari, and M. S. Feld, Phys. Rev. Lett. 73, 3375 (1994).

[99] F. S. Choa, M. H. Shih, J. Y. Fan, G. J. Simonis, P.-L. Liu, T. Tanbun-Ek, R. A. Logan, W. T. Trang, and A. M. Sergent, Appl. Phys. Lett. 67, 2777 (1995).

[100] D. L. Huffaker, J. Shin, and D. G. Deppe, Appl. Phys. Lett. 66, 1723 (1995).

[101] H. Taniguchi, H. Tomisawa, and J. Kido, Appl. Phys. Lett. 66, 1578 (1995).

[102] Z. Feit, M. McDonald, R. J. Woods, V. Archambault, and P. Mak, Appl. Phys. Lett. 68, 738 (1996).

[103] V. Sandoghdar, F. Treussart, J. Hare, V. Lefèvre-Seguin, J.-M. Raimond, and S. Haroche, Phys. Rev. A 54 R1777 (1996).

[104] S. Strauf, K. Hennessy, M. T. Rakher, Y.-S. Choi, A. Badolato, L. C. Andreani, E. L. Hu, P. M. Petroff, and D. Bouwmeester, Phys. Rev. Lett. 96, 127404 (2006). 
[105] S. Noda, Science 314, 260-261 (2006).

[106] X. Hachair, R. Braive, G. L. Lippi, D. Elvira, L. Le Gratiet, A. Lemaitre, I. Abram, I. Sagnes, I. Robert-Philip, and A. Beveratos, Phys. Rev. A 83, 053836 (2011).

[107] A. E. Siegman, Lasers (University Science Books, Mill Valley, CA, 1986).

[108] X. Duan, Y. Huang, R. Agarwal, and C. M. Lieber, Nature 421, 241-245 (2003).

[109] P. B. Johnson and R. W. Christy, Phys. Rev. B 6, 4370-4379 (1972).
[110] E. D. Palik, Handbook of optical constants of solids (Academic, New York, 1985).

[111] S. E. Miller, Bell Sys. Tech. J. 48, 7, 2059 (1969).

[112] In Vitro Diagnostics to 2013 - Market Research, Market Share, Market Size, Sales, Demand Forecast, Market Leaders, Company Profiles, Industry Trends, Market study by http://www.freedoniagroup.com (2011), date of last visit: 18 February 2012.

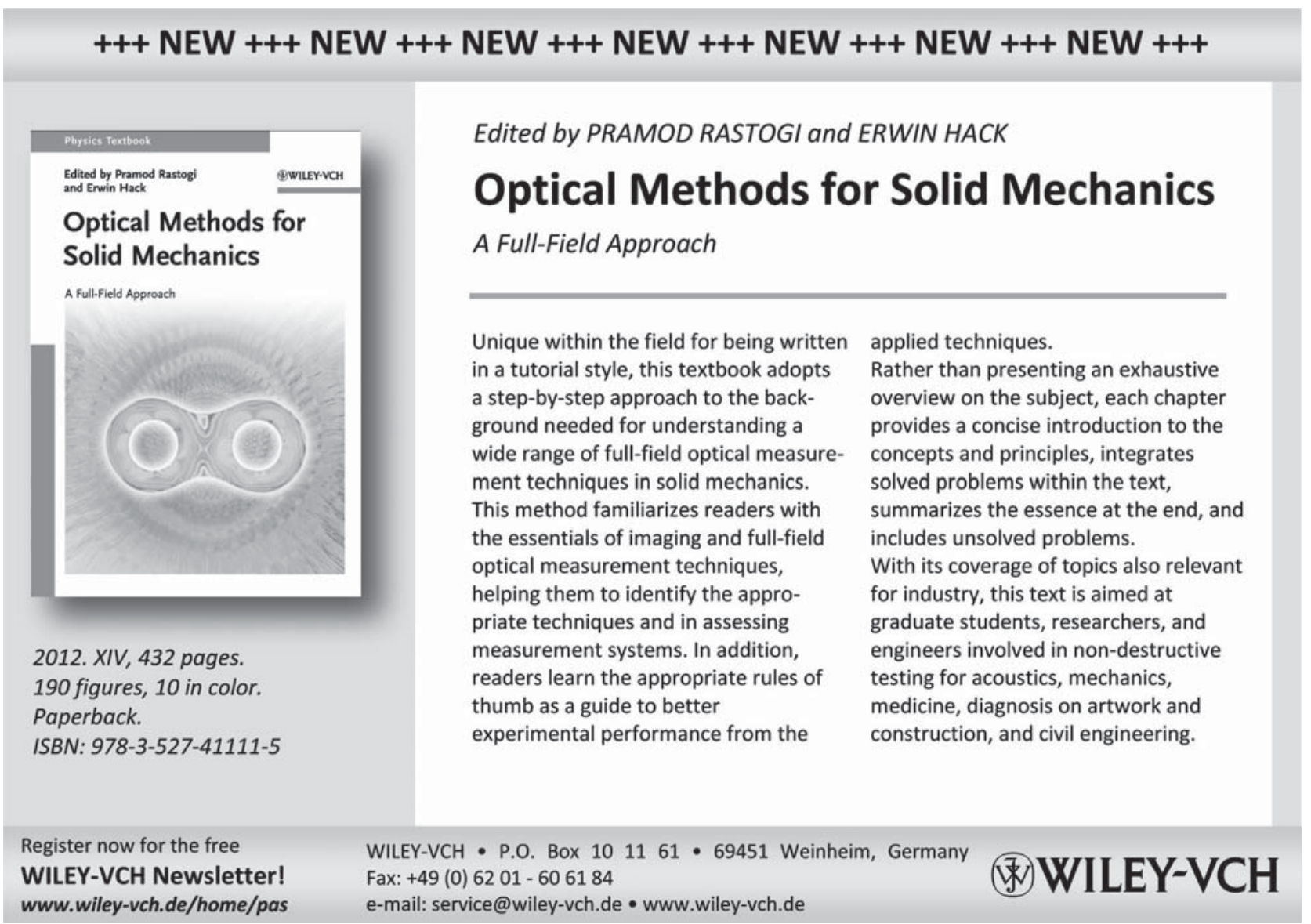

